\title{
Development of Monitoring Techniques for Laser Powder Bed Additive Manufacturing of Metal Structures
}

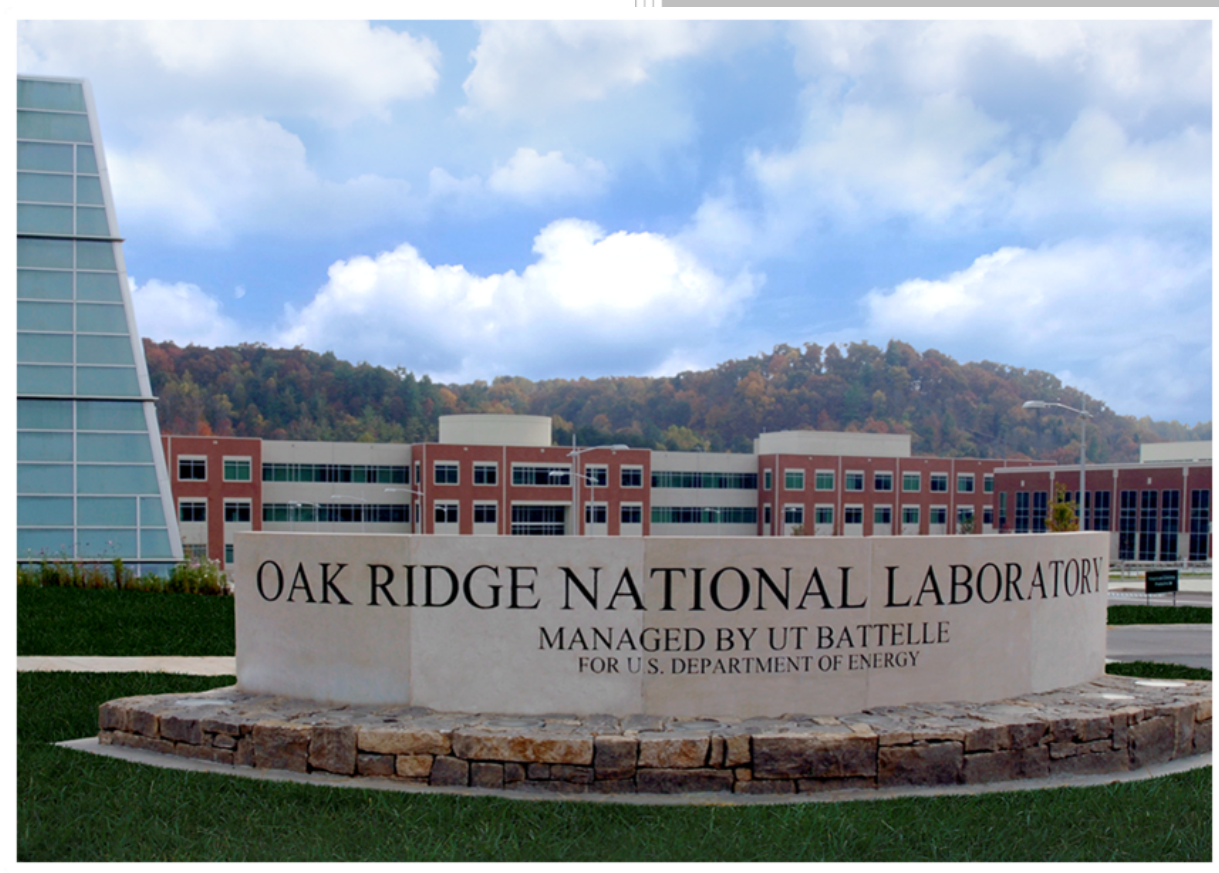

Luke Scime William Halsey James Haley Alka Singh Michael Sprayberry Amir Ziabari Vincent Paquit

September 2020

M3TC-20OR0403015 


\section{DOCUMENT AVAILABILITY}

Reports produced after January 1, 1996, are generally available free via US Department of Energy (DOE) SciTech Connect.

Website www.osti.gov

Reports produced before January 1, 1996, may be purchased by members of the public from the following source:

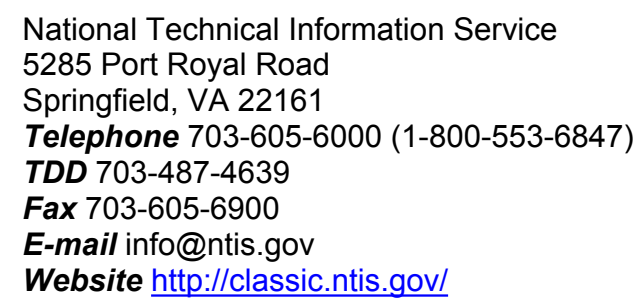

Reports are available to DOE employees, DOE contractors, Energy Technology Data Exchange representatives, and International Nuclear Information System representatives from the following source:

Office of Scientific and Technical Information

PO Box 62

Oak Ridge, TN 37831

Telephone 865-576-8401

Fax 865-576-5728

E-mail reports@osti.gov

Website http://www.osti.gov/contact.html

This report was prepared as an account of work sponsored by an agency of the United States Government. Neither the United States Government nor any agency thereof, nor any of their employees, makes any warranty, express or implied, or assumes any legal liability or responsibility for the accuracy, completeness, or usefulness of any information, apparatus, product, or process disclosed, or represents that its use would not infringe privately owned rights. Reference herein to any specific commercial product, process, or service by trade name, trademark, manufacturer, or otherwise, does not necessarily constitute or imply its endorsement, recommendation, or favoring by the United States Government or any agency thereof. The views and opinions of authors expressed herein do not necessarily state or reflect those of the United States Government or any agency thereof. 


\title{
DEVELOPMENT OF MONITORING TECHNIQUES FOR LASER POWDER BED ADDITIVE MANUFACTURING OF METAL STRUCTURES
}

\author{
Luke Scime \\ William Halsey \\ James Haley \\ Alka Singh \\ Michael Sprayberry \\ Amir Ziabari \\ Vincent Paquit
}

Date Published: September 2020

M3TC-20OR0403015

Prepared by

OAK RIDGE NATIONAL LABORATORY

Oak Ridge, TN 37831-6283

managed by

UT-BATTELLE, LLC

for the

US DEPARTMENT OF ENERGY

under contract DE-AC05-00OR22725 



\section{CONTENTS}

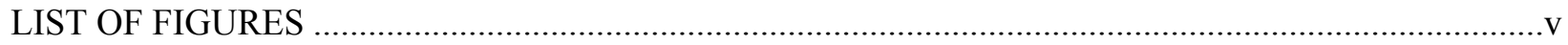

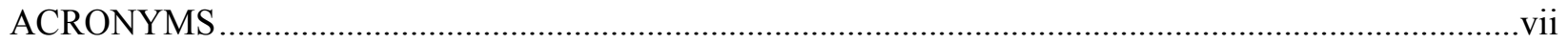

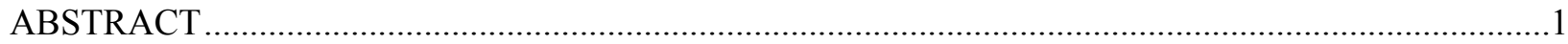

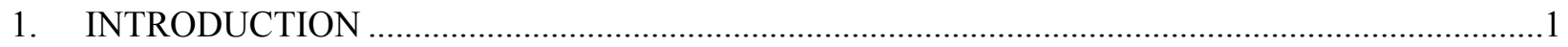

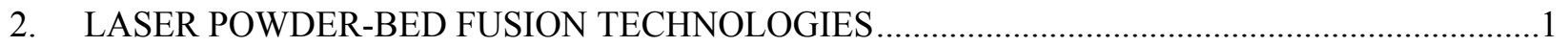

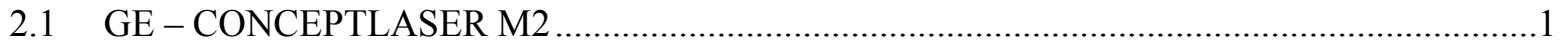

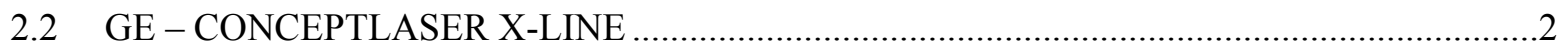

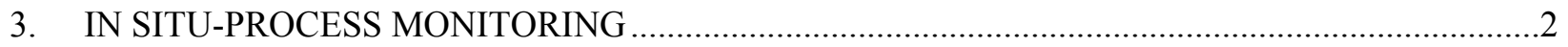

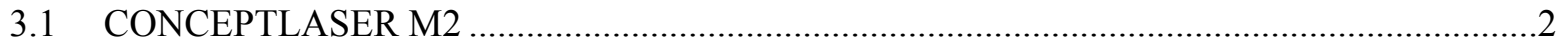

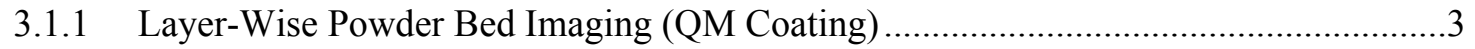

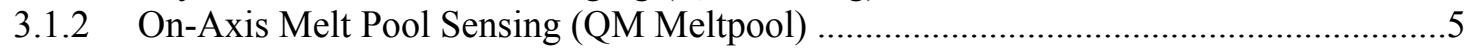

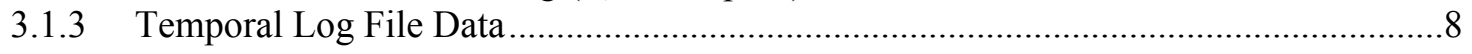

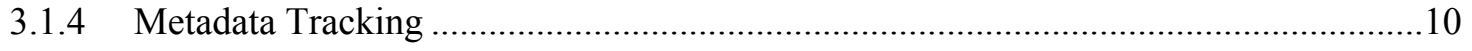

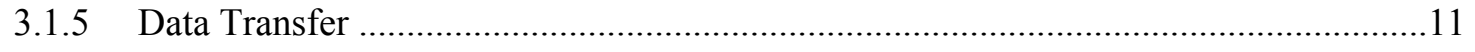

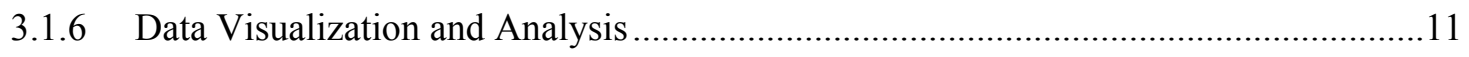

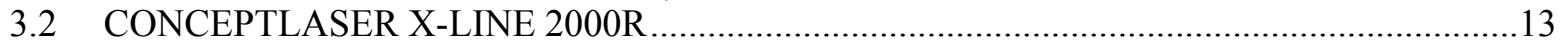

3.2.1 Layer-Wise Powder Bed Imaging (QM Coating) .................................................... 14

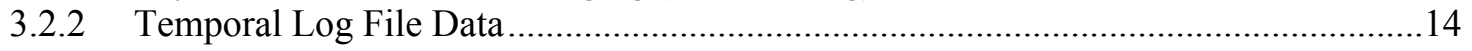

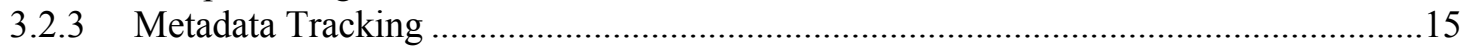

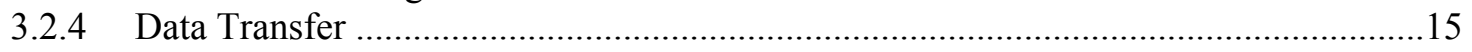

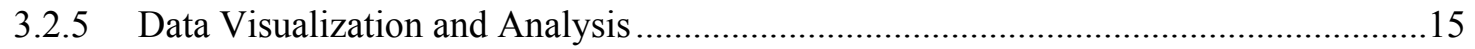

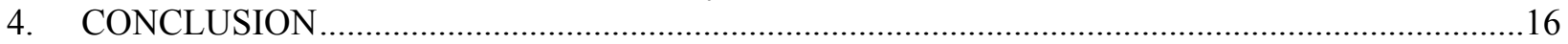

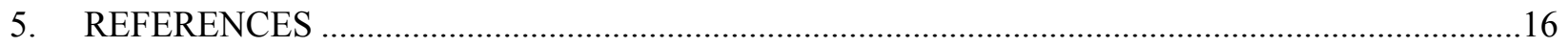

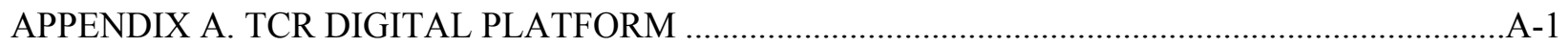

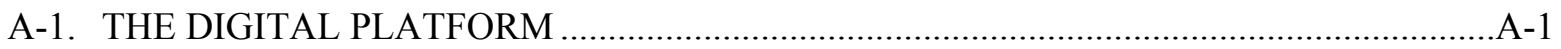

A-2. DATA STORAGE INFRASTRUCTURE AND DATABASE ARCHITECTURE ...............A-5

A-3. DIGITAL TOOL (WEB INTERFACE AND APIS) .....................................................A-5

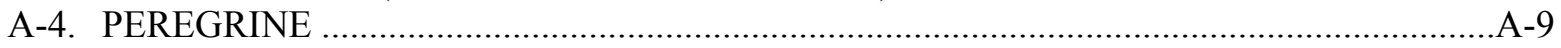

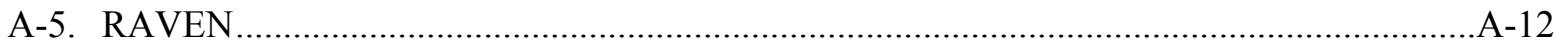

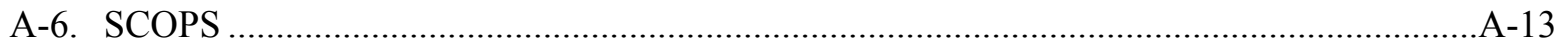

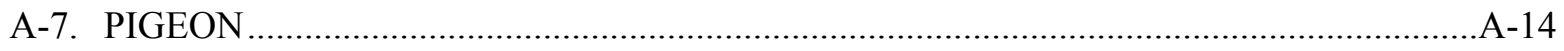

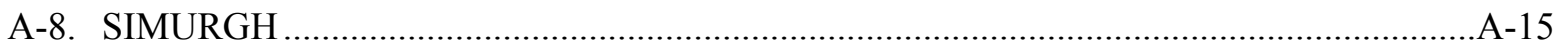

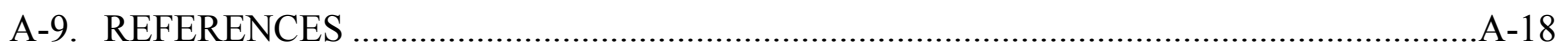





\section{LIST OF FIGURES}

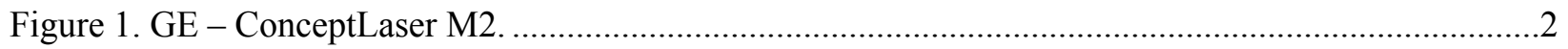

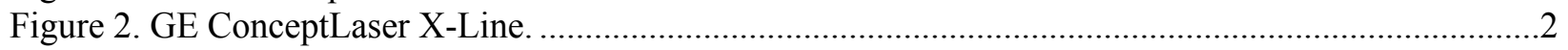

Figure 3. Exemplar imagery captured with the QM coating imaging system: (left) image taken

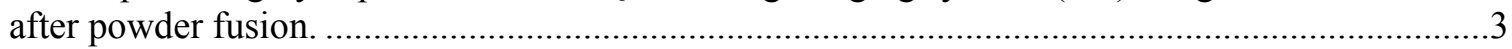

Figure 4. PhaseOne Machine Vision iXM-MV150F camera systems.....................................................

Figure 5. A test image captured using a $150 \mathrm{MP}$ camera on loan from the original equipment

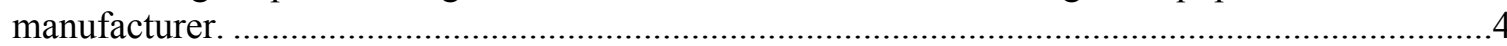

Figure 6. Duplicate hardware for the QM Meltpool system. ..................................................................

Figure 7. Representative on-axis photodiode data with an anomalous signature highlighted by a

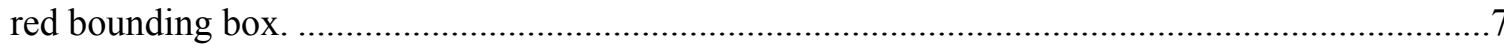

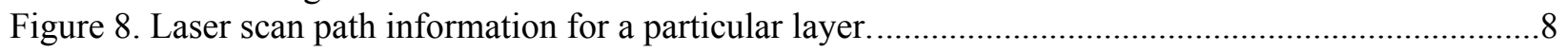

Figure 9. Example log file data displayed as a time series. ...........................................................

Figure 10. (left) Spatially mapped log file data from the top gas flow sensor; (right) Peregrinederived defect locations where blue voxels represent nominal material, and red voxels

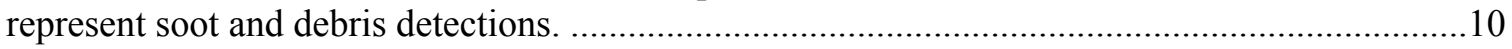

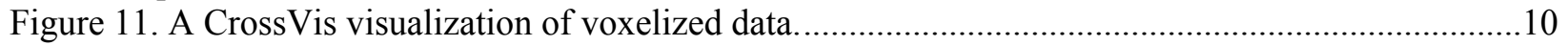

Figure 12. Anomaly detections overlaid on top of a post-fusion image of the powder bed......................11

Figure 13. Heat map (i.e., projection) of part swelling detections throughout the height of the

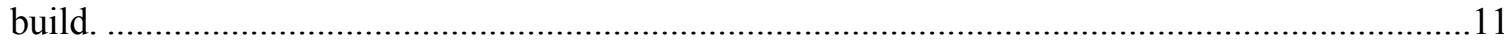

Figure 14. Print quality metrics reported for each pentagonal specimen...............................................12

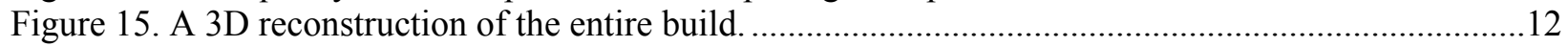

Figure 16. Relative shifts in the laser module positioning are apparent in different regions of the build, designated A, B, and C. Relative laser alignment shifts may be as much as $300 \mu \mathrm{m}$

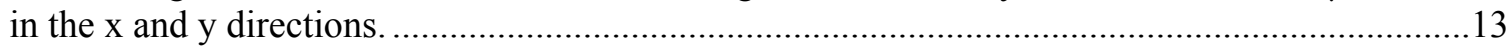

Figure 17. Time series visualization of soot detections, intended part cross section, and layer print

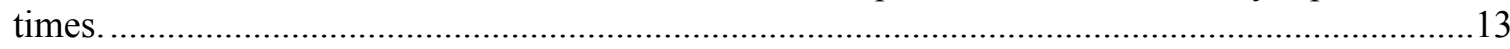

Figure 18. An image taken after powder fusion (left) and an image taken after powder spreading (right) ......................................................................................................................... 14

Figure 19. Example log file data displayed as a time series with a low-pass filter applied.......................15

Figure A-1. An overview of the digital platform.........................................................................

Figure A-2. A representation of an example digital thread. ............................................................

Figure A-3. A simplified digital workflow for the manufacture of a silicon carbide fuel element...........A-3

Figure A-4. Specimen numbering scheme following the ASTM 52900:2015€ standard [1].................A-4

Figure A-5. Representation of the microservices-based architecture utilized by the digital tool..............A-6

Figure A-6. An exemplar metadata entry form for an operation. ......................................................

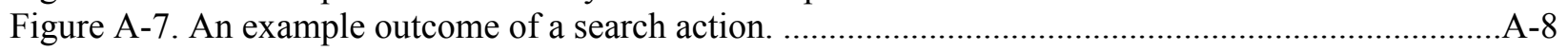

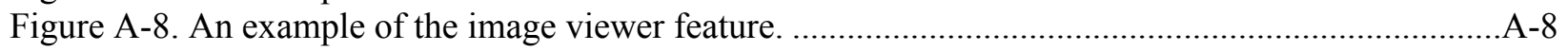

Figure A-9. Overview of Peregrine's capabilities and operation. ......................................................

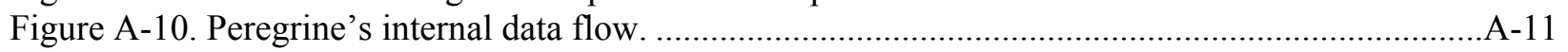

Figure A-11. (a) SCOPS imaging system, two stereo high-resolution visible cameras and an infrared camera, (b) visible trackable surface roughness of representative deposition, (c) infrared imaging showing thermal dissipation, (d) 3D map constructed from surface features, (e) tracked deformation induced from a combination of residual strain and thermal expansion from (f) 3D mapped infrared signal showing hot regions........................A-13

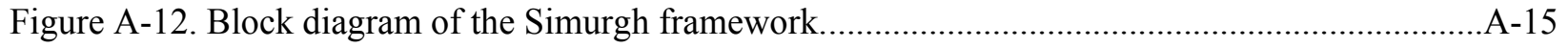

Figure A-13. Example test results on a synthetic data set. ..................................................................16

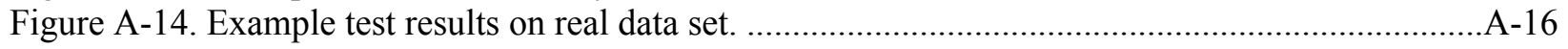





\section{ACRONYMS}

$\begin{array}{ll}\text { AI } & \text { augmented intelligence } \\ \text { AM } & \text { additive manufacturing } \\ \text { API } & \text { beam hardening } \\ \text { BH } & \text { computer-aided design } \\ \text { CAD } & \text { chemical vapor infiltration } \\ \text { CVI } & \text { directed energy deposition } \\ \text { DED } & \text { digital image correlation } \\ \text { DIC } & \text { deep learning } \\ \text { DL } & \text { DSCNN-Perceptron } \\ \text { DSCNN } & \text { uncorrected standard analytical } \\ \text { DSCNN-P } & \text { field programmable gate array } \\ \text { FDK } & \text { graphics processing unit } \\ \text { FPGA } & \text { Internet of Things } \\ \text { GPU } & \text { laser powder bed fusion } \\ \text { IoT } & \text { model-based iterative reconstruction } \\ \text { LPBF } & \text { Manufacturing Demonstration Facility } \\ \text { MBIR } & \text { machine learning } \\ \text { MDF } & \text { megapixel } \\ \text { ML } & \text { Oak Ridge National Laboratory } \\ \text { MP } & \text { standard triangle language } \\ \text { ORNL } & \text { Transformational Challenge Reactor } \\ \text { STL } & \text { Technology Innovation Program } \\ \text { TCR } & \text { x-ray computed tomography } \\ \text { TIP } & \\ \text { XCT } & \\ & \end{array}$





\begin{abstract}
The Transformational Challenge Reactor (TCR) program is leveraging additive manufacturing (AM) technologies to fabricate nuclear grade components to be assembled into a fully functional microreactor core. Compared to traditional manufacturing technologies, additive manufacturing technologies allow (1) observation of the manufacturing process at a much higher resolution in real time using in situ monitoring technologies to capture the sensor signature that scientifically describes each event occurring over time and space, and (2) validation of the manufacturing process quality using domain-informed data analytics techniques as a potential qualification and certification methodology for the final component.
\end{abstract}

This report provides an update on the program work on laser powder bed fusion in-situ process monitoring and associated data analytics results. Examples are provided to illustrate the progress. Elements of the Digital Thread and data management are discussed in the main document, and an extensive supplemental material section is provided detailing the Digital Platform, as well as its implementation and components. In conclusion the path forward for the next fiscal year is discussed.

\title{
1. INTRODUCTION
}

The FY19 TCR report Monitoring for Additive Manufacturing Technologies: Report on Progress, Achievements and Limitations of Monitoring Techniques establishes that the implementation and deployment of the Digital Platform requires three elements: (1) a hardware architecture for data exchange and storage, (2) a software platform for advanced data analytics, and (3) an information-rich manufacturing and material database for domain discovery. The previous report also provides an evaluation of the existing sensing modalities installed on selected additive manufacturing (AM) machines and an overview of image processing results. Based on these preliminary results, a path forward is established to improve the Digital Platform through enhancement of the hardware and software components.

This report provides an update of the envisioned implementation for laser powder bed fusion (LPBF) technology. A companion report will discuss monitoring of the binder jet additive manufacturing process also utilized by the TCR program. This document is structured as follows: the first section gives a brief overview of the two LPBF machines, explaining the manufacturing process, the dimensions of the build chamber, and the type of instrumentation provided on the systems. The second section discusses the insitu monitoring results in FY20, addressing both hardware and software components. All results are illustrated with examples of data collection and processing results, followed by the conclusion.

Note that several images included in this document are from parts not produced for the Transformational Challenge Reactor (TCR). This choice was due to alleviate export control concerns and produce a document that is publicly available.

\section{LASER POWDER-BED FUSION TECHNOLOGIES}

\subsection{GE - CONCEPTLASER M2}

The ConceptLaser M2 system (Figure 1) is a metal printer using LPBF technology to manufacture parts. It has a build volume of $250 \times 250 \times 350 \mathrm{~mm}^{3}$ and is equipped with two $400 \mathrm{~W}$ lasers which are used for melting a stack of thin layers of powder that describes the $3 \mathrm{D}$ geometries. The maximum laser velocity is $7.5 \mathrm{~m} / \mathrm{s}$. Depending on geometry, material, and process parameters this system offers a maximum build rate volume of $20 \mathrm{~cm}^{3} / \mathrm{h}$. The manufacturing process is accomplished in four steps: 
1. Each layer is prepared with a specific printing scan strategy and associated process parameters such as laser power and speed.

2. A raking mechanism covers the build plate with a layer of powder.

3. The melting process programmed during step 1 is applied to the layer

4. Once the layer is complete, the stage suporting the build plate lowers to the desired layer thickness (typically 20 to $80 \mu \mathrm{m}$ ).

This process repeats until completion of the geometry. This system is equipped with standard sensing technologies to ensure correct mechanical operation. Additionally, two optional technologies are available to assess the quality of the melting process (QM Meltpool) and to assess the quality of the layer of powder (QM Coating).

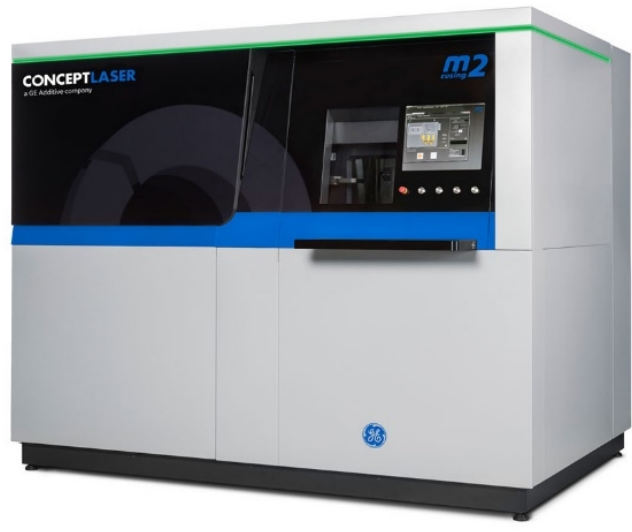

Figure 1. GE - ConceptLaser M2.

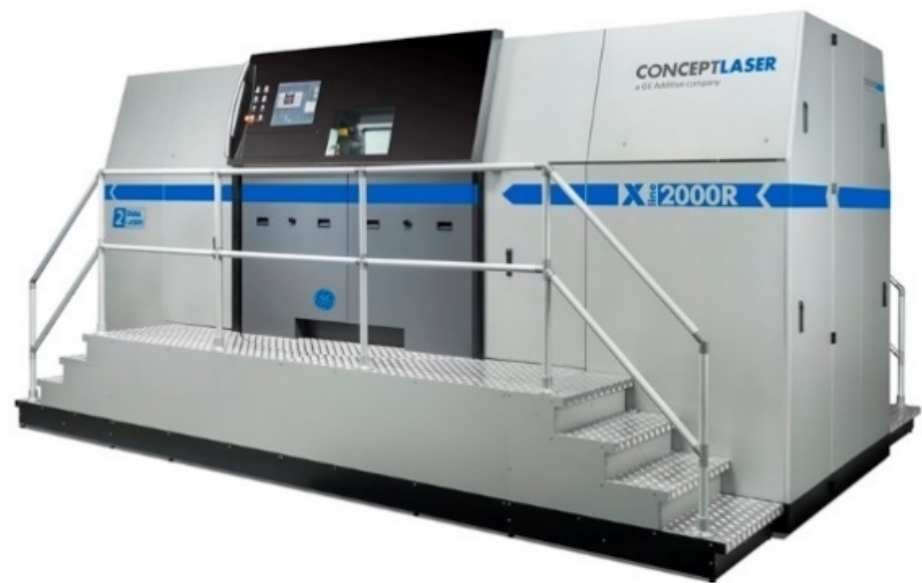

Figure 2. GE ConceptLaser X-Line.

\subsection{GE - CONCEPTLASER X-LINE}

The ConceptLaser X-Line system (Figure 2) uses the same technology and manufacturing process as the M2 system. However, the X-Line has a build volume of $800 \times 400 \times 500 \mathrm{~mm}^{3}$ (about 7.5 times the volume of the M2) and uses two 1,000 W lasers. The maximum laser velocity is $7.5 \mathrm{~m} / \mathrm{s}$. Depending on geometry, material and process parameters, this system offers a build rate volume of $120 \mathrm{~cm}^{3} / \mathrm{h}(6$ times the build rate of the M2). The X-Line also offers a partial implimentation of the QM Coating system; however, less than half of the build plate can be monitored.

\section{IN SITU-PROCESS MONITORING}

\subsection{CONCEPTLASER M2}

A single ConceptLaser M2 LPBF printer is located at the Manufacturing Demonstration Facility (MDF) at Oak Ridge National Laboratory (ORNL), within the export control area of the high bay. This machine is internally designated as the ConceptLaserM2-ORNL1, and it produces several in-situ data streams which are collected and analyzed as part of the TCR program. The following subsections describe each of these data streams, highlighting any changes in hardware, software, or analysis methodologies in FY20. The in-situ data are analyzed and visualized together using the Peregrine software package, which is described in the Digital Platform supplementary material. 


\subsubsection{Layer-Wise Powder Bed Imaging (QM Coating)}

A 5 megapixel (MP) 16-bit grayscale camera, sensitive in the visible spectrum, captures an image of the entire print area immediately after powder fusion and powder spreading of each layer. A top-mounted flash module provides illumination. This system was installed and is maintained by ConceptLaser. Examples of post-fusion and post-spreading images for the same layer of a build are shown in Figure 3.
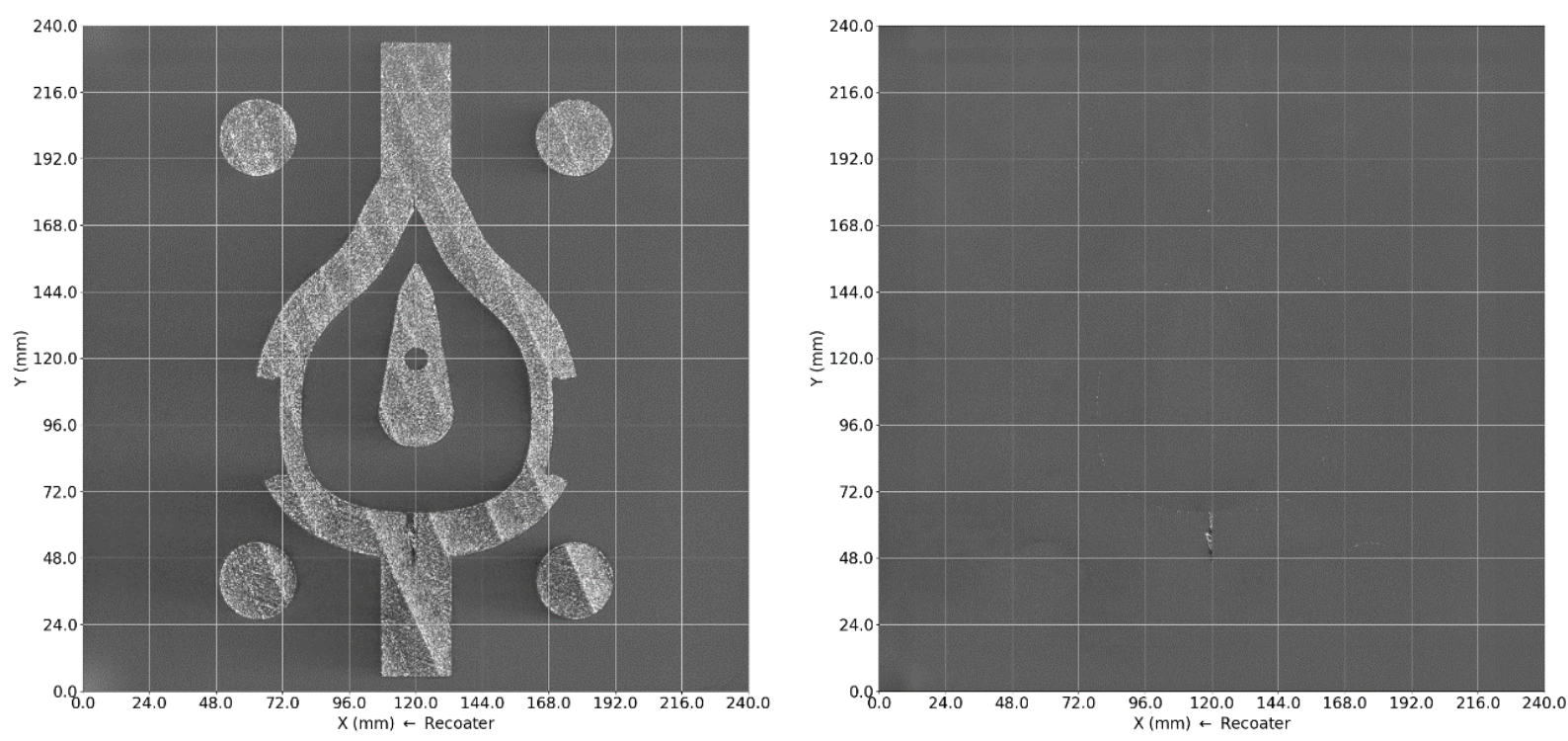

Figure 3. Exemplar imagery captured with the QM coating imaging system: (left) image taken after powder fusion. The unmelted powder region appears in a darker shade of gray compared to the printed region; (right) image taken after powder spreading. The previous printed layer should be covered by powder at this point. Note that a small region in the bottom-center indicates a protuberance from the previous layer.

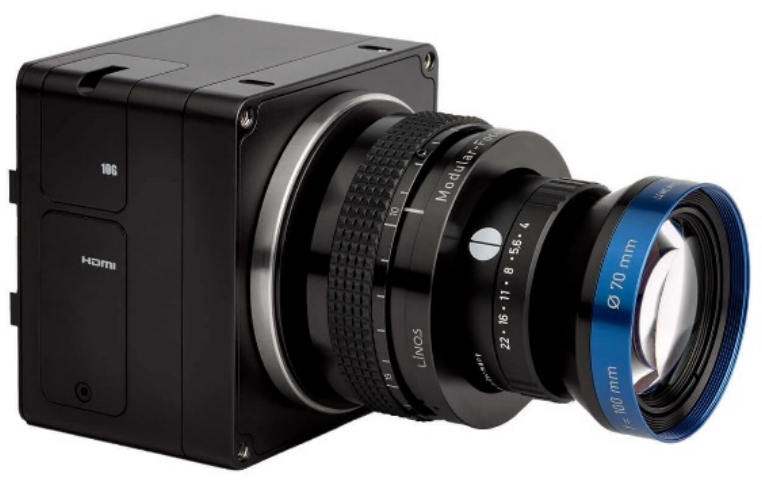

Figure 4. PhaseOne Machine Vision iXM-MV150F camera systems. 
This imaging system has been used since FY19, but an upgrade to a PhaseOne 150 MP camera is planned for FY21 (see Figure 4) to improve the effective resolving power of the system from $\sim 300$ to $\sim 40 \mu \mathrm{m}$.

This resolving power improvement is required to properly monitor the build quality of thin-wall structures $(<1 \mathrm{~mm})$ which are expected to be printed for the reactor core components. Figure 5 shows an example image captured by the $150 \mathrm{MP}$ camera on a test bed.

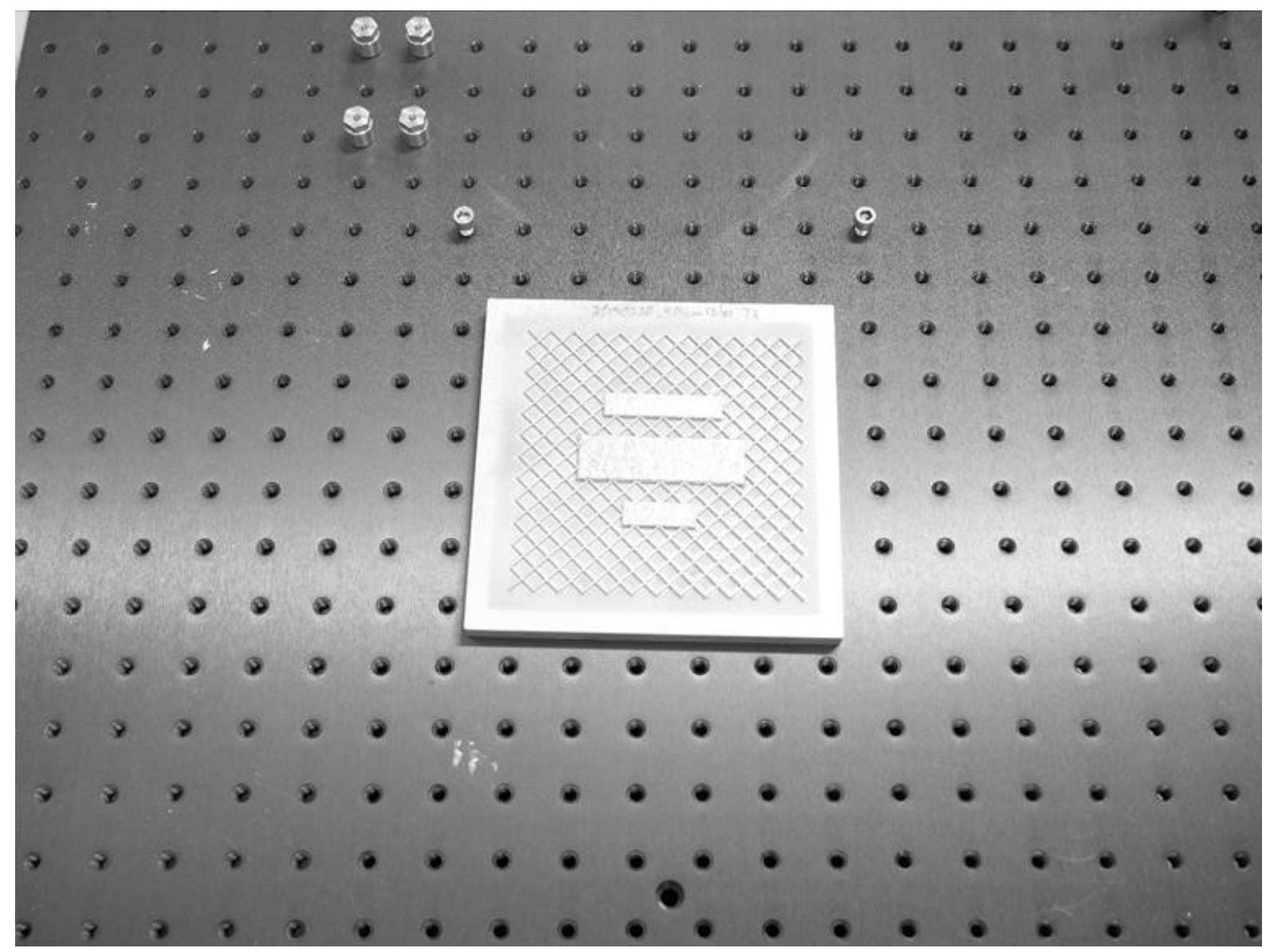

Figure 5. A test image captured using a 150 MP camera on loan from the original equipment manufacturer.

Peregrine's neural network analysis of these powder bed images enables the detection of several types of defects and process flaws [1], as well as comparisons between the as-printed and intended geometries. Detectable anomalies include damage to the recoating mechanism, improper powder spreading, part geometry swelling or distortion, damage to the as-printed components, abnormal generation of spatter and other melt pool ejecta, and improper powder fusion. Improving the camera's resolving power is expected to enable improved detection of spatter particles, recoater streaking, and fusion defects in thin-walled structures.

For comparison to the intended geometry, computer-assisted design (CAD) information is first converted into a standard triangle language (STL) format and is then "sliced" into layer images using the Arcam Build Assembler software. Work on an alternative ORNL-developed slicing software was started in FY20 and will continue in FY21. Note that the accuracy of the geometrical comparisons is limited by the resolving power of the camera, as well as the registration error between the camera data and the geometry data. 


\subsubsection{On-Axis Melt Pool Sensing (QM Meltpool)}

The ConceptLaser QM Meltpool system consists of a photodiode and a high-speed camera which are coaxially aligned with each laser module such that they continually observe the molten pool throughout the build. Nominally, this system is installed and maintained by ConceptLaser and is duplicated for each of the machine's two laser modules. Throughout FY20, however, research was conducted to improve the efficacy of this system. This research necessitated several changes to the ConceptLaser sensing hardware, and several additional changes are planned for FY21.

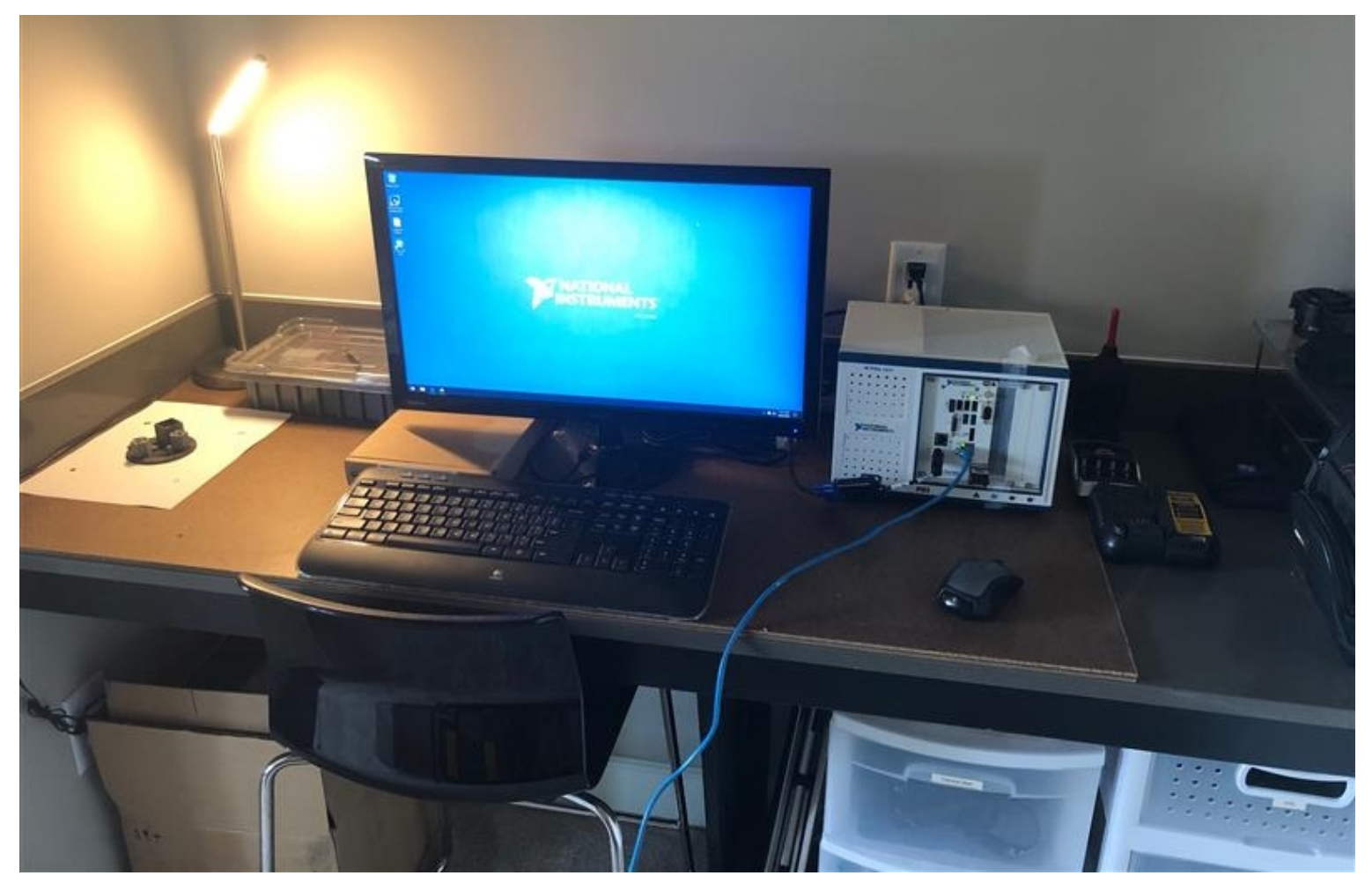

Figure 6. Duplicate hardware for the QM Meltpool system.

As installed by the original equipment manufacturer, the on-axis photodiode captures light intensity data in the region around the melt pool at a rate of $10-100 \mathrm{kHz}$ and is sensitive in the $350-1,100 \mathrm{~nm}$ range. Extensive testing revealed that the overwhelming majority of the sensor's signal can be attributed to reflected laser light at $1,060 \mathrm{~nm}$. This is not ideal, as the thermal emissions from the molten pool and the just-solidified material are much more likely to be correlated with subsurface porosity, and other salient printing defects, than reflected laser light. Therefore, ORNL modified the photodiode system on Laser Module 1 as follows:

a. A high pass filter was installed, modifying the sensitivity range to 350-1042 nm and thus blocking out a larger percentage of the reflected laser light.

b. The photodiode was replaced with a similar model with a larger detector area; this allows for more light emission from the just-solidified material to be captured by the sensor.

c. The relative gain (sensitivity) of the sensor was increased from 60 to $70 \mathrm{~dB}$.

A critical software bug (specifically an overflow error) was identified in the ConceptLaser QM Meltpool software. This bug caused the sensor to saturate artificially, dramatically limiting its dynamic range.

While ConceptLaser has not fixed this issue, ORNL software was created to recover the saturated values, 
thereby allowing full use of the sensor's dynamic range. Several other artifacts are present in the QM Meltpool data. Most of these artifacts can be removed by existing hardware upgrades; however, ConceptLaser has yet to allow ORNL to purchase and install this hardware. In the interim, the artifacts are being removed, albeit with lower fidelity, using post-processing software. Figure 7 shows an example of the QM Meltpool photodiode data after the described ORNL hardware and software modifications. The signature within the red box appears to correlate with the part distortion visible in the powder bed images shown in Figure 3. The blue circle and annulus are imaging artifacts which cannot currently be removed from the data. Spatial mapping and artifact removal of the photodiode data are performed by Peregrine.

The on-axis visible-light camera continuously calculates the number of pixels in the region around the melt pool with light intensities above an arbitrary global threshold at a rate of 10-100 kHz. To date, data from this sensor have not been used for TCR, as the camera system has not functioned correctly for either laser module at any point since the ConceptLaser M2 machine was delivered to ORNL. In FY20, equipment was purchased to duplicate portions of the ConceptLaser QM Meltpool system (Figure 6). In FY21, this equipment will be used to develop custom melt pool morphology classification algorithms similar to those described by Scime et al. [2]. These algorithms will be deployed on a real-time field programmable gate array (FPGA) system. This will provide ORNL with an alternative on-axis melt pool monitoring system, bypassing the original equipment manufacturer's software and some of the hardware, and allowing for development of improved defect detection capabilities. 


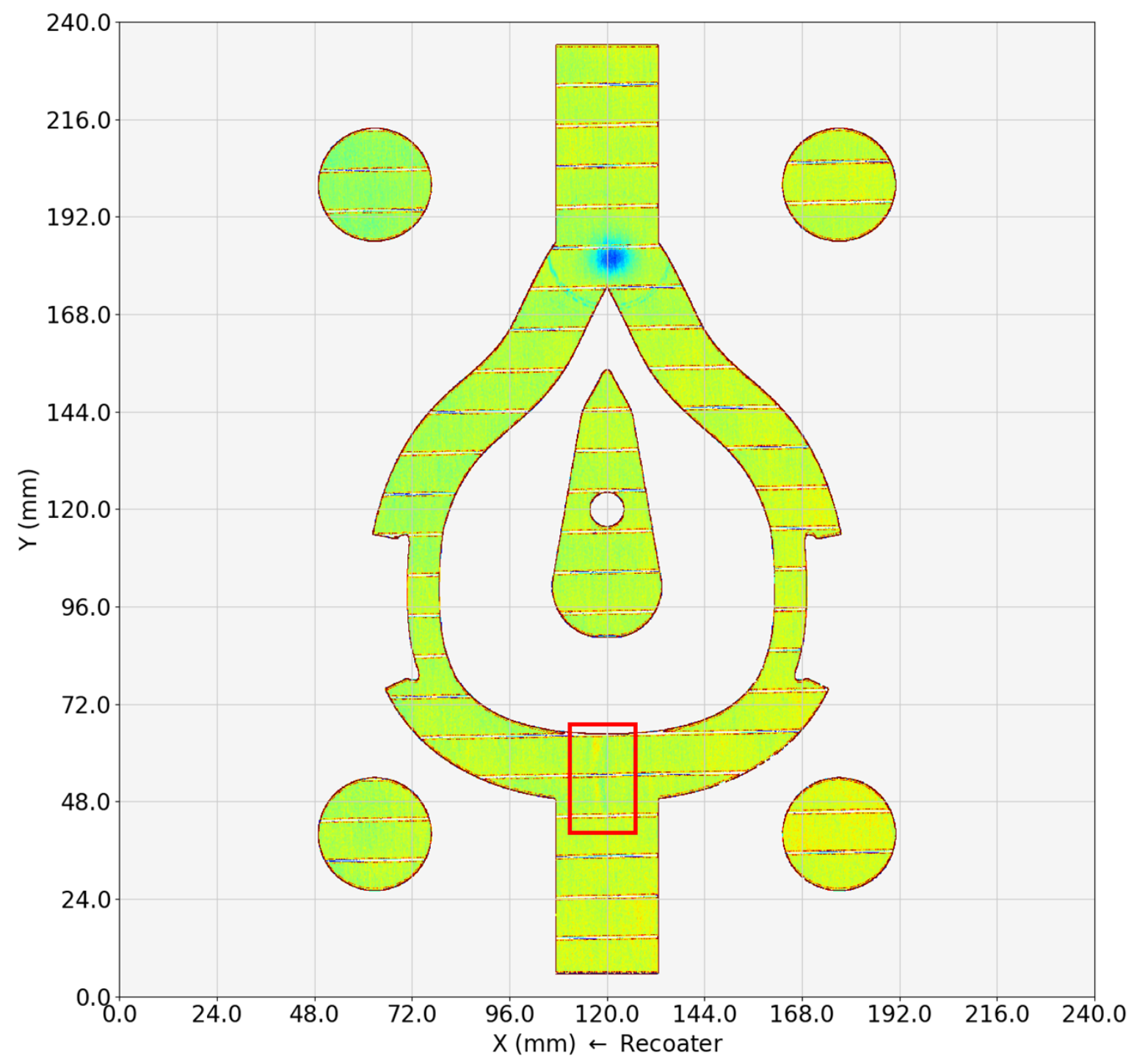

Figure 7. Representative on-axis photodiode data with an anomalous signature highlighted by a red bounding box.

The QM Meltpool system also indirectly provides information concerning the laser scan path. This information is spatially mapped by Peregrine as shown in Figure 8. Because the laser scan strategy is known to influence defect formation mechanisms in LPBF processes, it is critical to provide this scan information to AM process engineers and designers. Knowledge of the scan strategy also allows for the spatial mapping of other temporal data, as discussed in the following subsection. 


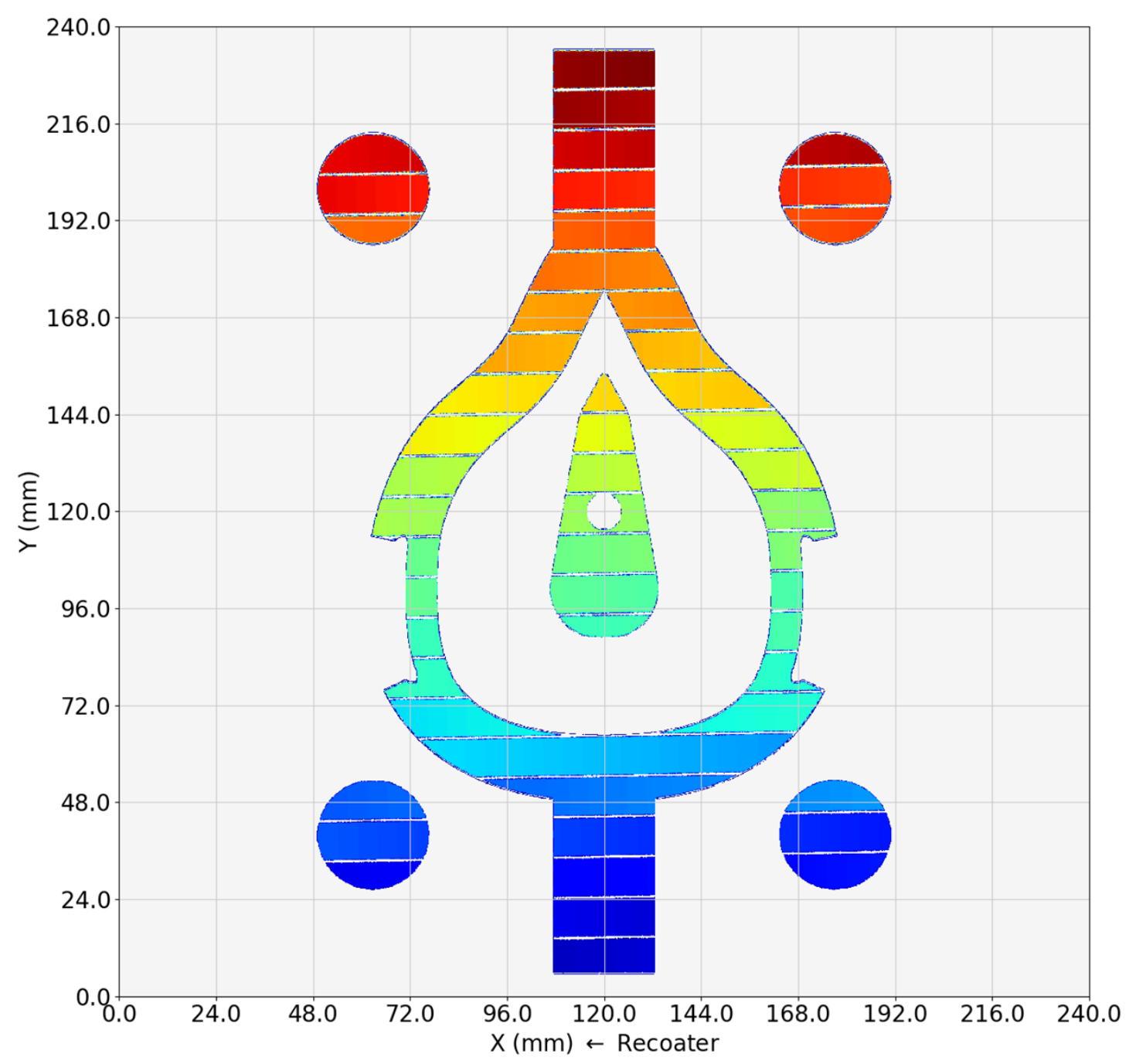

Figure 8. Laser scan path information for a single layer. Darker blue regions were scanned first, and darker red regions were scanned last.

\subsubsection{Temporal Log File Data}

At the end of each build, a log file is produced which reports various machine error states, as well as temporal sensor streams, including build chamber gas (argon) flow rates, build chamber oxygen concentrations, build plate temperature, and the temperatures of selected components in the laser optic trains. This system is installed and maintained by ConceptLaser, and the data can be viewed using Peregrine, as shown in Figure 9. 


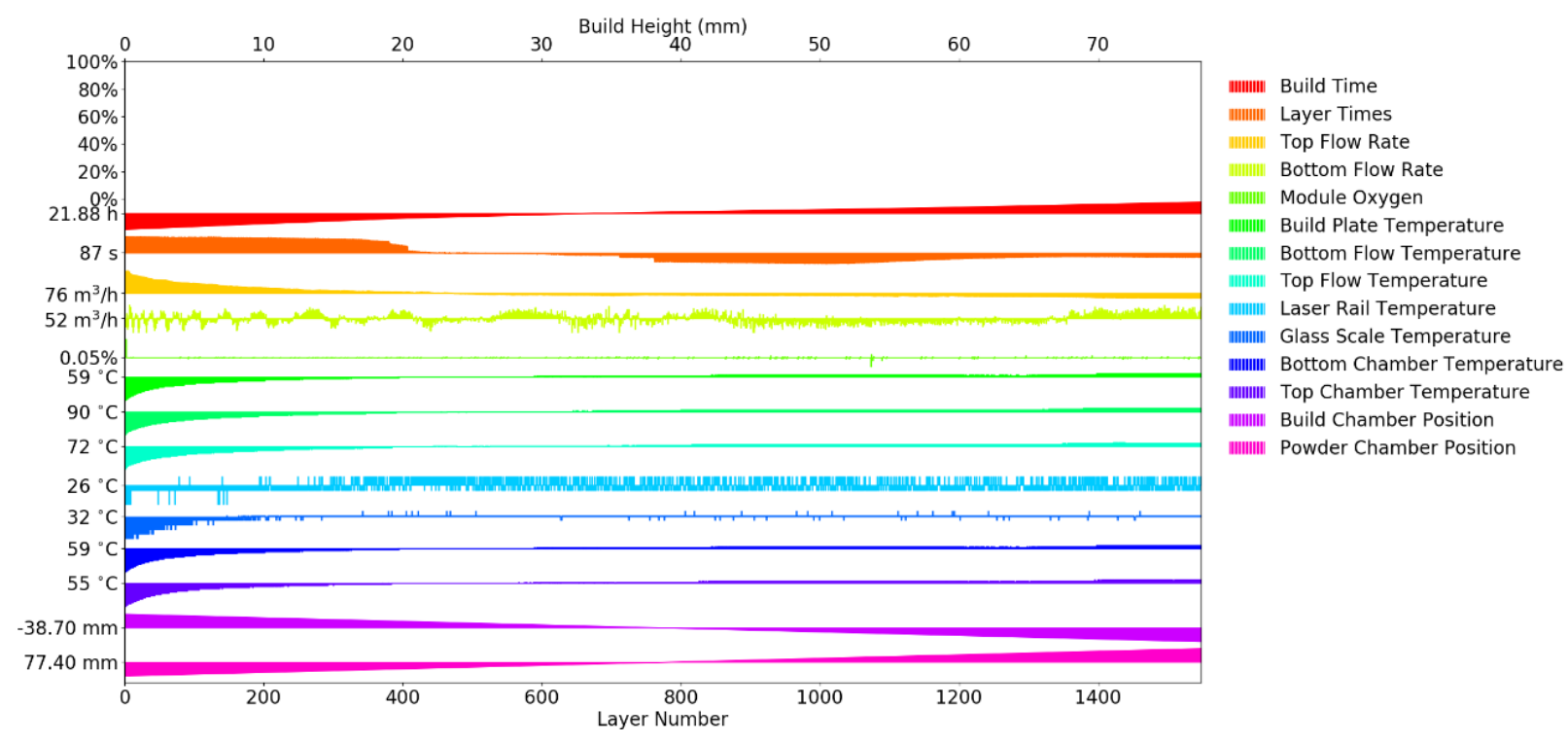

Figure 9. Example log file data displayed as a time series.

In FY20, preliminary work has demonstrated the ability to spatially map the temporal log file data using the laser scan path information indirectly provided by the QM Meltpool system. The goal of this operation is to convert the temporal data into a format where it may be directly correlated with detected defects from the in-situ layer-wise powder bed images. To this end, points along the scan path are sampled in order to determine the set of pixels (from in-situ imaging) that each scan line intersects. This process assigns an approximate timestamp to each voxel location - allowing the temporal log file data to be mapped onto the voxelized representation of the build volume. The mapped data are taken from measurements that are taken during the fusion phase of each layer, during which changes in the environment may affect the melt pool and subsequent cooling conditions of the part. Spatially mapping these data may enable correlation of printed part defects to process anomalies such as changes in oxygen concentration or fluctuations in the shield gas flow rate.

Visualizing the data spatially allows for qualitative assessment of the consistency of the build, as well as visually relating changes in the sensor data to detected defects, as shown in Figure 10. ORNL has previously demonstrated these types of correlations for the Arcam electron beam powder bed fusions systems, and work in this area for the ConceptLaser M2 will continue in FY21. 

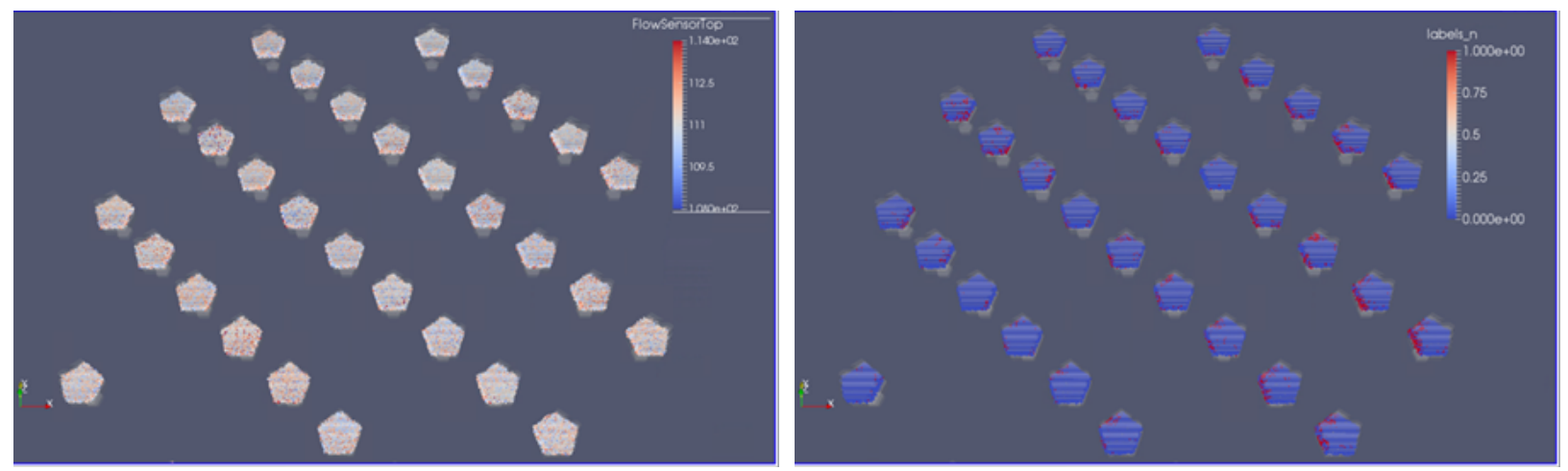

Figure 10. (left) Spatially mapped log file data from the top gas flow sensor; (right) Peregrine-derived defect locations where blue voxels represent nominal material, and red voxels represent soot and debris detections.

The mapped and voxelized data may then be visualized and further analyzed using tools such as CrossVis [3], a tool developed at ORNL outside of the TCR program, as shown in Figure 11. This facilitates quantitative correlation of defects to log file and scan path data.

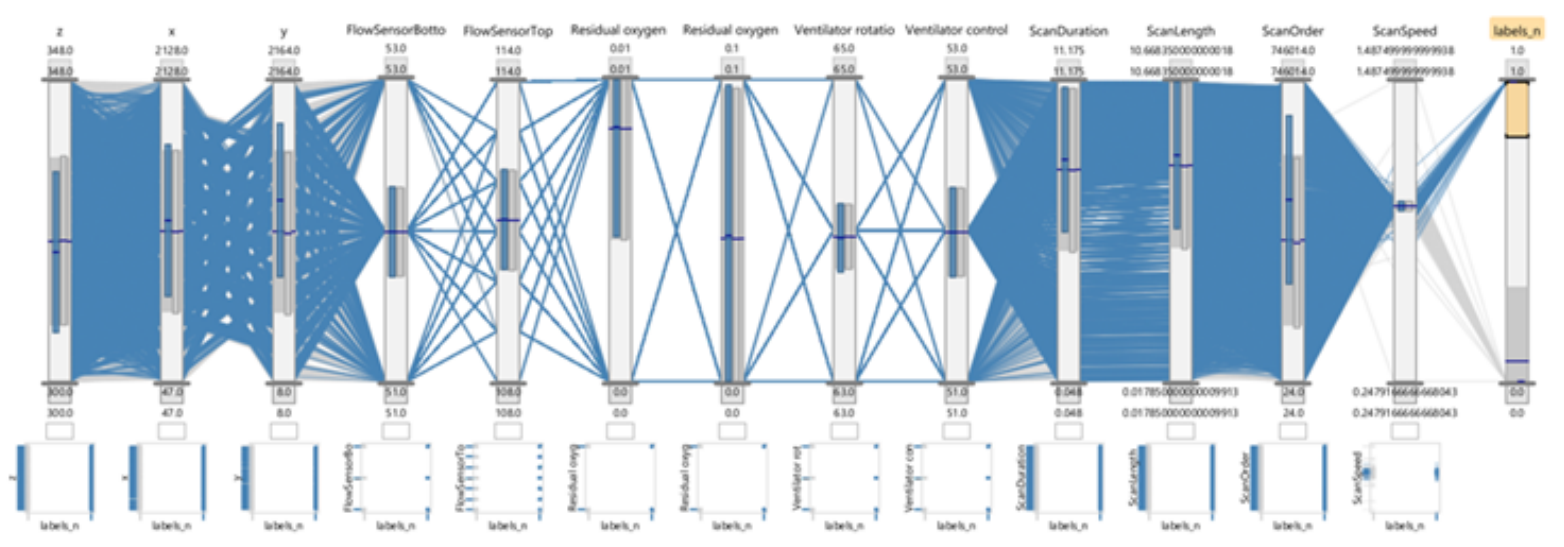

Figure 11. A CrossVis visualization of voxelized data. This investigation allows for quantitative correlations between the sensor data, scan path data, and Peregrine-predicted defects

\subsubsection{Metadata Tracking}

Build-specific metadata are tracked using a combination of Peregrine and the Digital Tool web interface that was newly developed in FY20. Tracked metadata now include the build name, build start date, build end date, various processing parameters, data sensitivity levels (e.g., export control restrictions), project and customer information, feedstock material type, feedstock batch information, printer operator information, ambient environmental data, and various operator-input notes. In FY21, the Digital Tool's capabilities will continue to be expanded, allowing for increased data search functionality and the ability to view Timelines of feedstock batch utilization, as well as printer calibration and maintenance histories as they relate to each build. Images of the entire build continue to be taken in a photobooth using a handheld camera. New in FY20, these post-build images, along with any images taken during the build or of any handwritten operator notes, are viewable from the Peregrine interface. 


\subsubsection{Data Transfer}

Currently, the in-situ process data are manually recovered from the ConceptLaser M2 using an encrypted external hard drive following each build. This hard drive is then hand-delivered to one or more desktop computers located in the TCR office space, and from there, the data are uploaded to the Data Storage Server. Metadata are tracked using both Peregrine and the digital tool web interface. Work in FY21 will explore connecting the ConceptLaser M2 directly to a secure Ethernet network to enable real-time data analysis and automated data upload during each build. This capability has been demonstrated for several other printers at ORNL.

\subsubsection{Data Visualization and Analysis}

The in-situ data produced by the ConceptLaser M2 are analyzed by trained machine operators and data team members using the Peregrine software package. A total of 62 ConceptLaser M2 builds were analyzed in FY20. A broader overview of Peregrine is provided in the Digital Platform supplementary material. To demonstrate the usage of Peregrine in the context of the ConceptLaser M2, a case study involving a single build of nominally identical pentagonal test specimens is discussed below.

Peregrine uses a neural network to analyze and classify each pixel in every layer of powder bed imaging data. One way to visualize these results is by overlaying the classifications on top of the powder bed images as shown in Figure 12. These classifications can be cumulatively analyzed throughout the entirety of the build and visualized as heat maps. Figure 13 shows a heat map highlighting the prevalence of insitu part distortion (i.e., swelling) in each pentagonal specimen. These results show that swelling is most common in certain printed corner geometries.

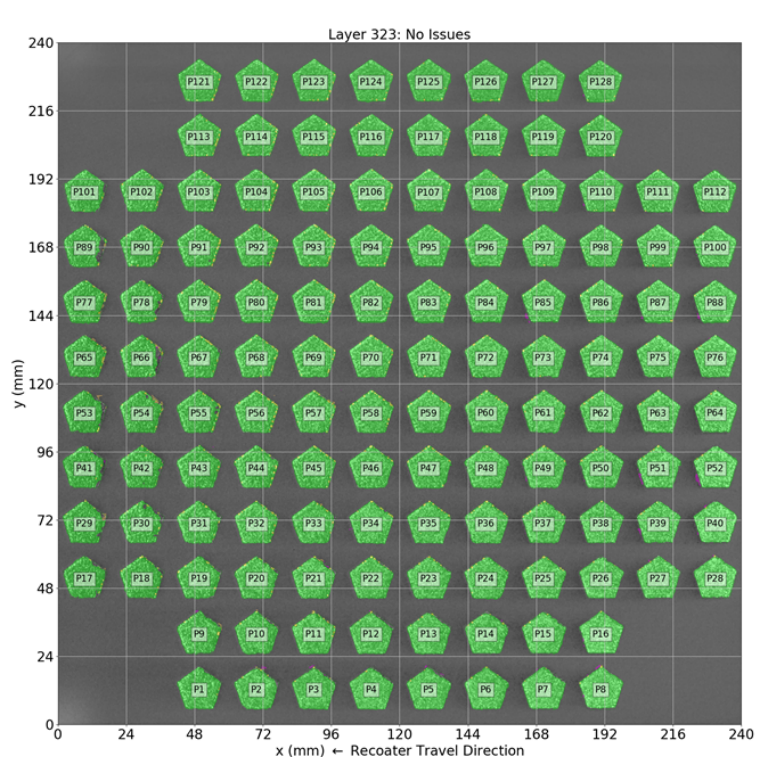

Figure 12. Anomaly detections overlaid on top of a post-fusion image of the powder bed.

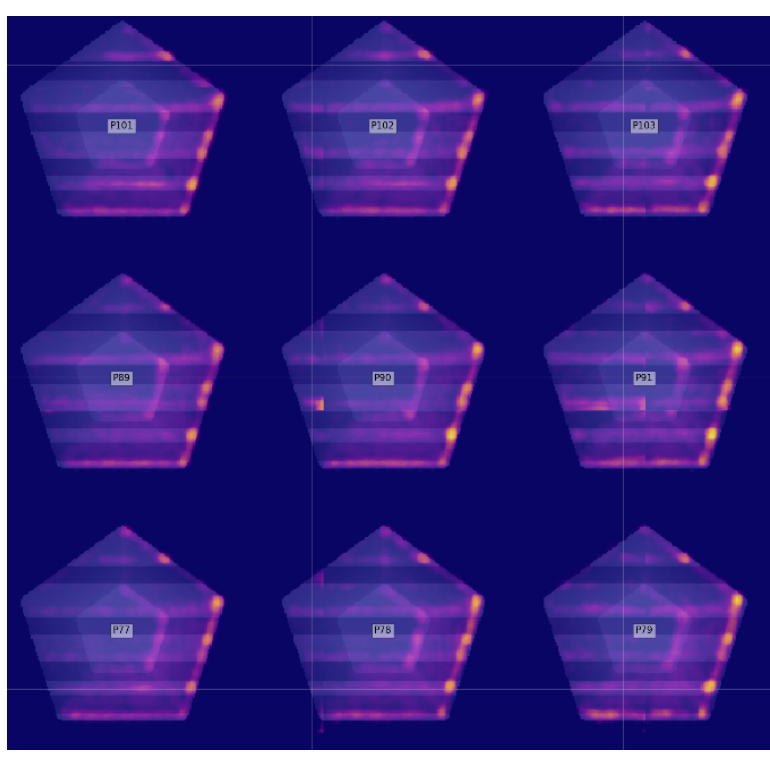

Figure 13. Heat map (i.e., projection) of part swelling detections throughout the height of the build.

Statistics summarizing the pixel-wise anomaly detections for each part are also reported. Figure 14 shows a print "quality" score reported for each part, and it is immediately evident that parts printed differently in different regions of the powder bed. Similar observations can also be made by viewing a 3D reconstruction of the build data (Figure 15). Note that the correlation of these summary statistics to part 
properties and performance metrics is ongoing and is discussed in a separate FY20 TCR report titled Viability of Data Analytics to Ascertain Component Performance for Additive Manufacturing.
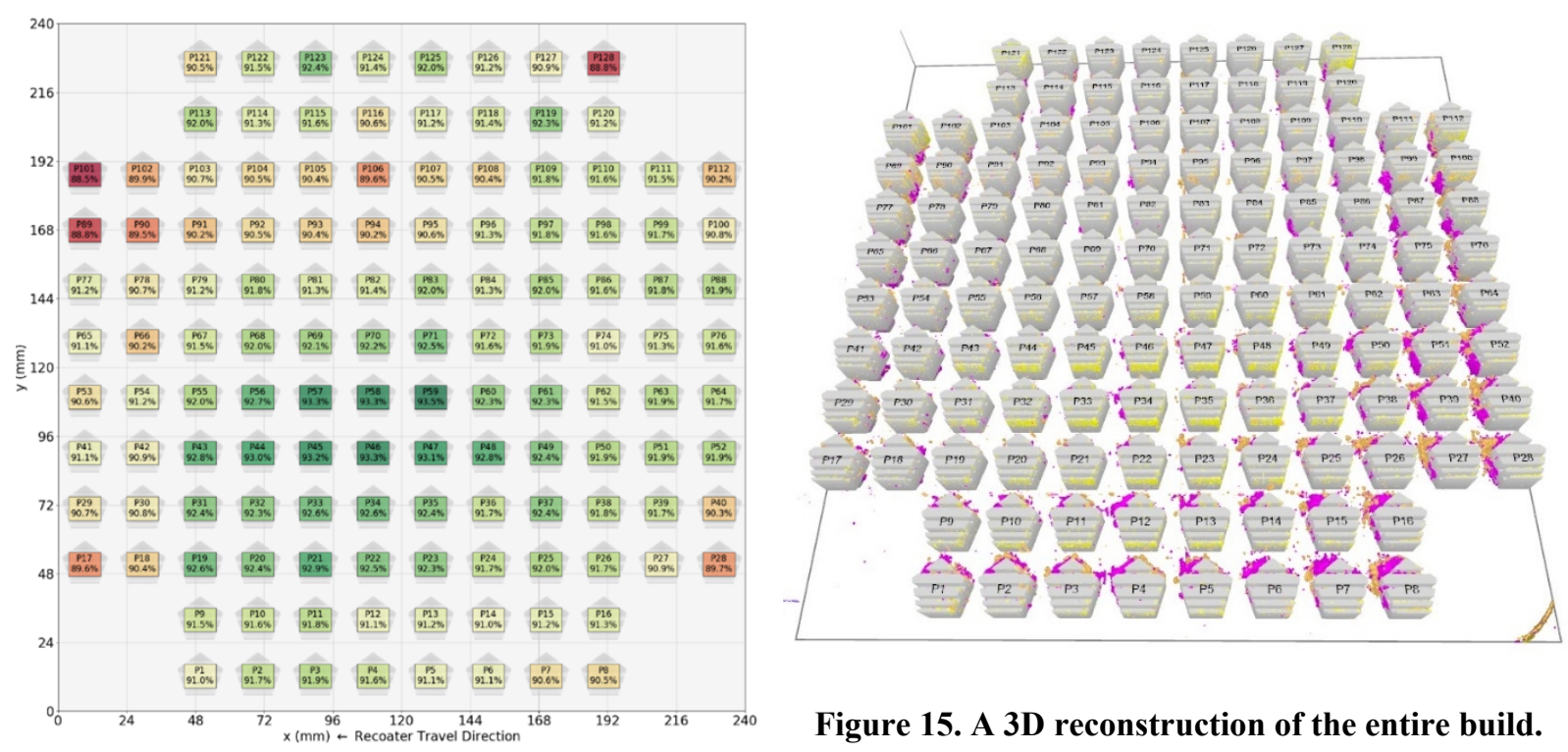

Figure 14. Print quality metrics reported for each pentagonal specimen.

In this example build, the laser module used to fuse the specimens was alternated throughout the duration of the build. As reported in FY19, Peregrine is able to quantify the relative offsets between the laser systems, as shown in Figure 16. Viewing the anomaly detections temporally makes it clear that different laser modules also result in different amounts of spatter generation. At layer 273 (Figure 17), the amount of "soot" detected increases as the laser module switches. Interestingly, this also corresponds with a drop in the layer print time, even though the cross-sectional geometry of the pentagons remained constant. 

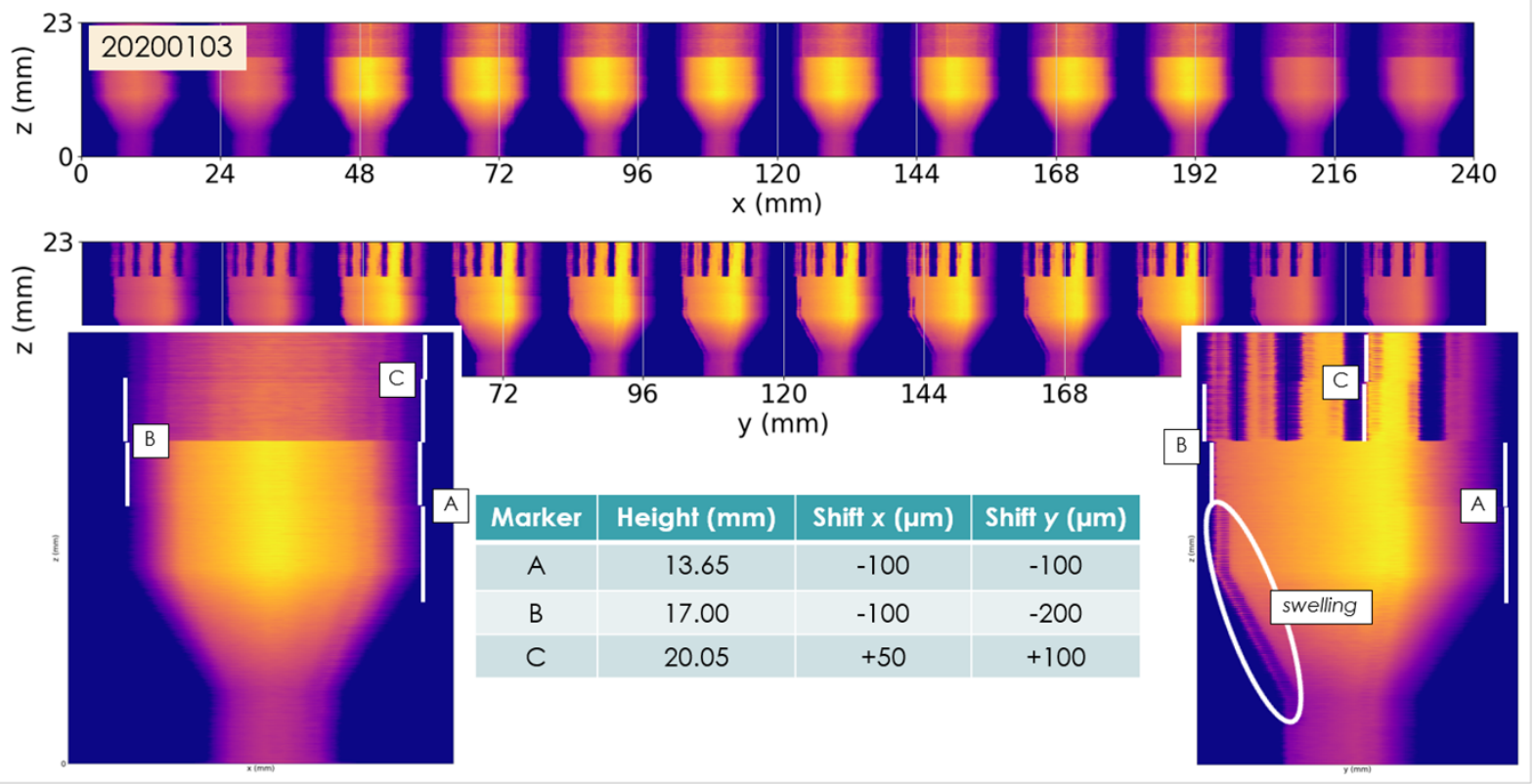

Figure 16. Relative shifts in the laser module positioning are apparent in different regions of the build, designated $\mathrm{A}, \mathrm{B}$, and $\mathrm{C}$. Relative laser alignment shifts may be as much as $300 \mu \mathrm{m}$ in the $\mathrm{x}$ and $\mathrm{y}$ directions.

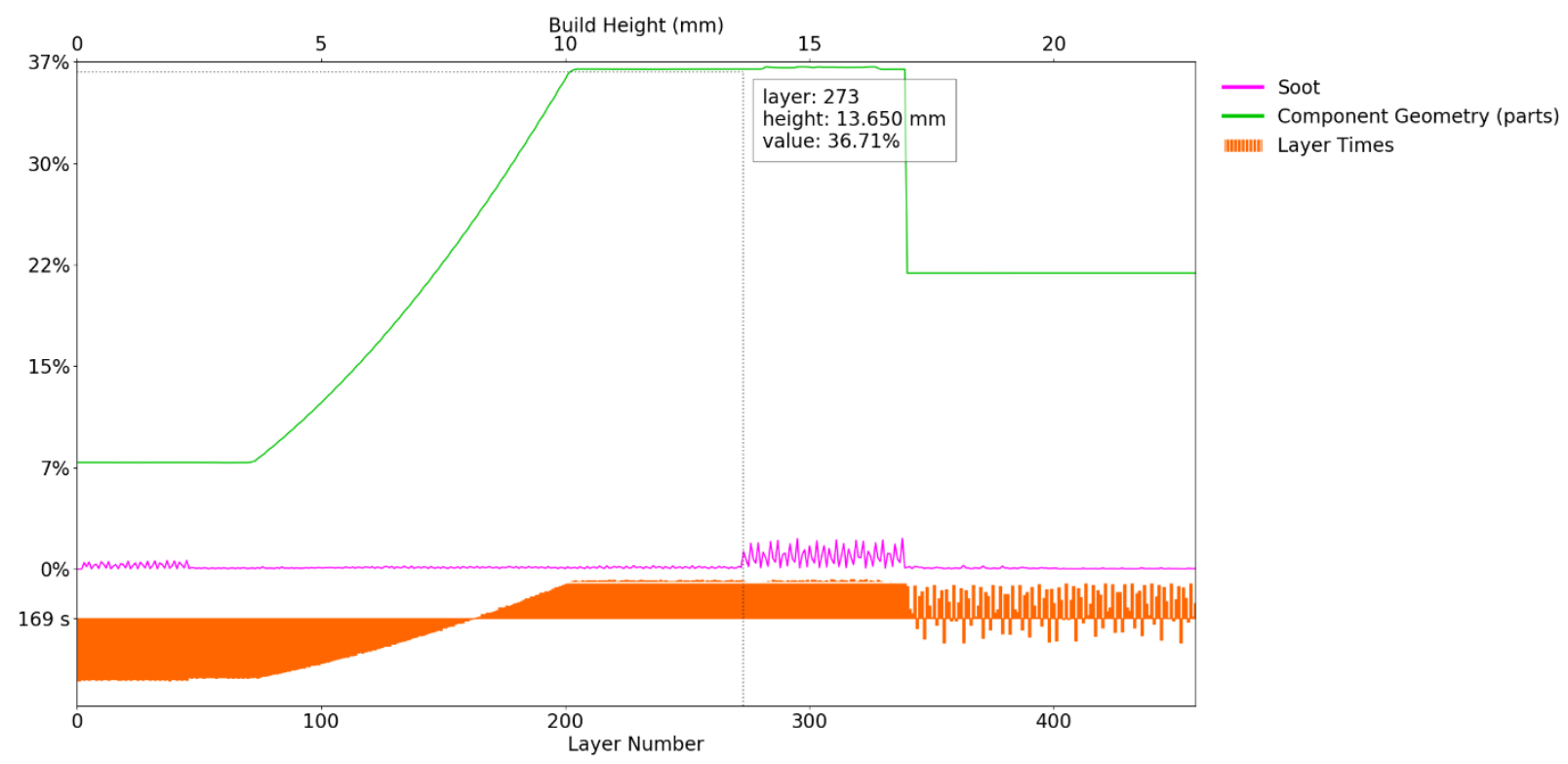

Figure 17. Time series visualization of soot detections, intended part cross-section, and layer print times.

\subsection{CONCEPTLASER X-LINE 2000R}

A single ConceptLaser M2 X-Line 2000R L-PBF printer is located at the MDF within the export control area of the high bay. This machine is internally designated ConceptLaserXLine2000R-ORNL1, and it 
produces several in-situ data streams which are collected and analyzed as part of the TCR program. The following subsections describe each of these data streams, highlighting any changes in hardware, software, or analysis methodologies in FY20. The in-situ data are analyzed and visualized together using the Peregrine software package, which is described in the Digital Platform supplementary material.

\subsubsection{Layer-Wise Powder Bed Imaging (QM Coating)}

A 12 MP 8-bit grayscale camera, sensitive in the visible spectrum, captures an image of approximately $30 \%$ of the print area immediately after powder fusion and powder spreading for each layer. Sidemounted lighting provides illumination. This system was installed and is maintained by ConceptLaser. However, layer-wise data capture is managed by ORNL via Peregrine. Examples of post-fusion and postspreading images for the same layer of a build are shown in Figure 18.
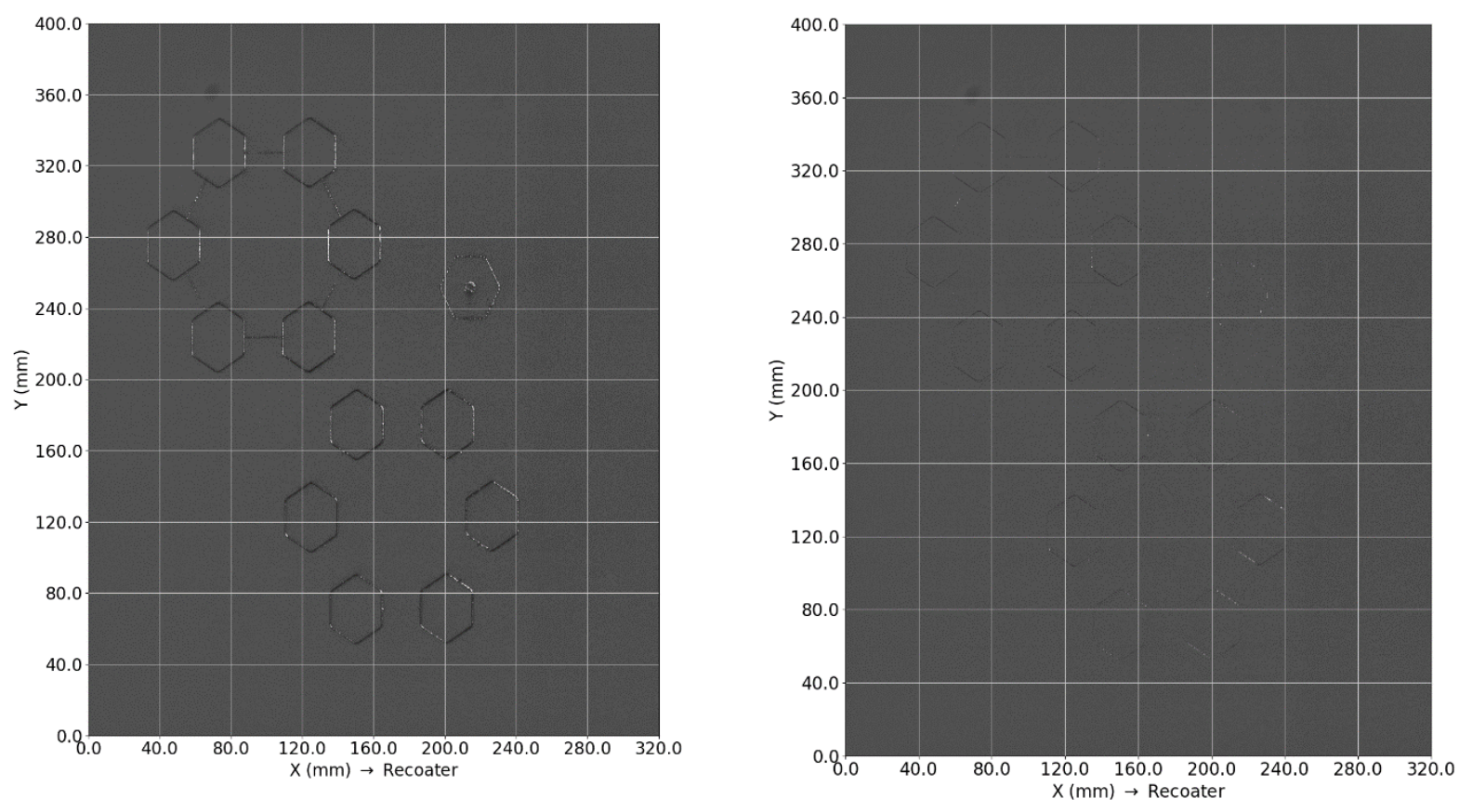

Figure 18. An image taken after powder fusion (left) and an image taken after powder spreading (right).

Initial testing of this imaging system was performed at the end of FY19, but full data capture capabilities were not completed until FY20. Peregrine performed neural network analysis of these powder bed images, enabling the detection of several types of defects and process flaws [1]. Detectable anomalies include damage to the recoating mechanism, improper powder spreading, part geometry swelling or distortion, damage to the as-printed components, and abnormal generation of spatter and other melt pool ejecta. Note that comparison of the as-printed geometry to the intended geometry is not possible until an appropriate calibration target is printed and imaged on this system.

\subsubsection{Temporal Log File Data}

At the end of each build, a log file is produced which reports various machine error states, as well as temporal sensor streams, including build chamber gas (argon) flow rates, build chamber oxygen concentrations, build plate temperature, and the temperatures of selected components in the laser optic trains. This system is installed and maintained by ConceptLaser, and the data can be viewed using 
Peregrine, as shown in Figure 19. Additional work in FY20 was performed to enable the filtering of these, sometimes noisy, data streams.

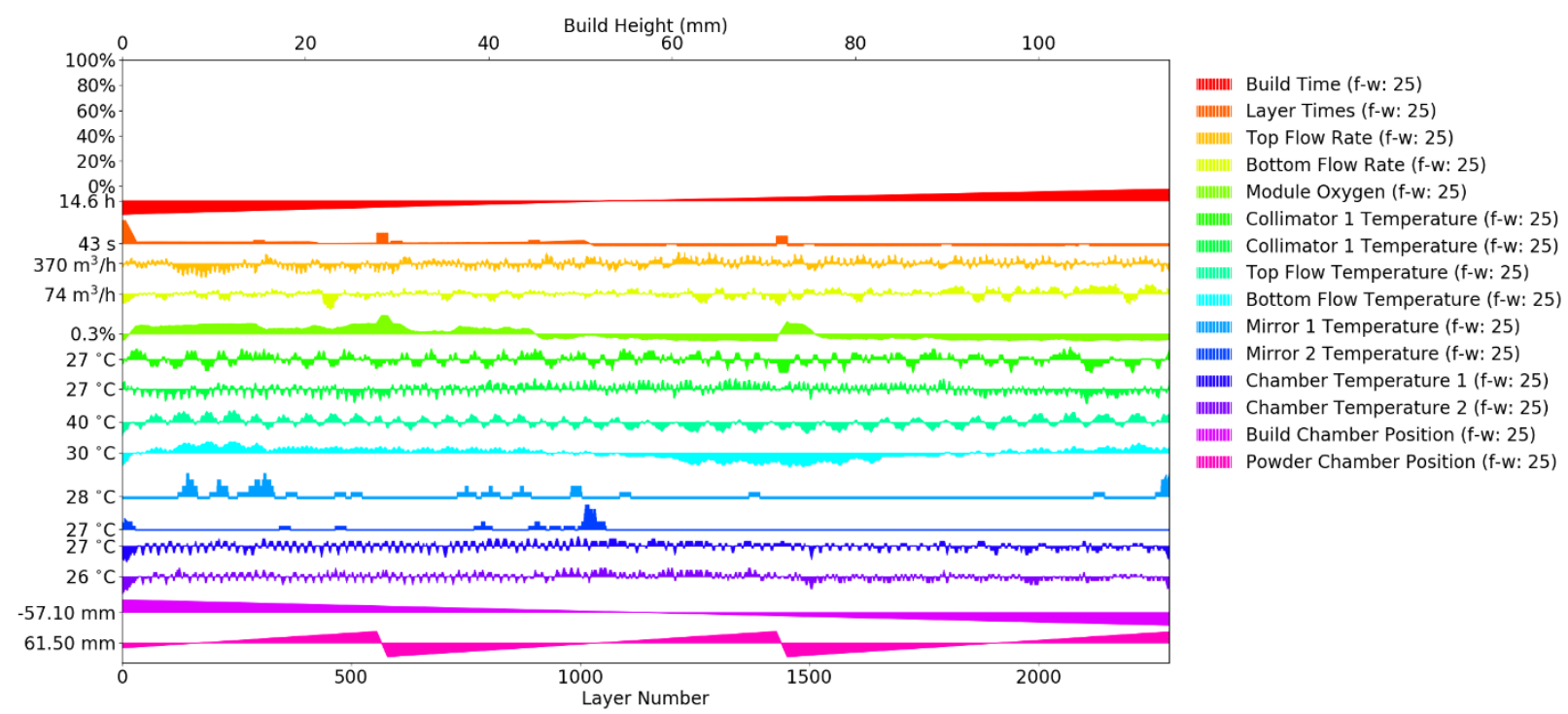

Figure 19. Example log file data displayed as a time series with a low-pass filter applied.

\subsubsection{Metadata Tracking}

Build-specific metadata are tracked using a combination of Peregrine and the Digital Tool web interface that was newly developed in FY20. Tracked metadata now include the build name, build start date, build end date, various processing parameters, data sensitivity levels (e.g., export control restrictions), project and customer information, feedstock material type, feedstock batch information, printer operator information, ambient environmental data, and various operator-input notes. In FY21, the digital tool's capabilities will continue expanding, allowing for increased data search functionality and the ability to view Timelines of feedstock batch utilization, as well as printer calibration and maintenance histories as they relate to each build. Images of the entire build continue to be taken in a photobooth using a handheld camera. New in FY20, these post-build images, along with any images taken during the build or of any handwritten operator notes, are viewable from the Peregrine interface.

\subsubsection{Data Transfer}

Currently, the log file data are manually recovered from the ConceptLaser X-Line 2000R using an encrypted external hard drive following each build. This hard drive is then hand-delivered to one or more desktop computers located in the TCR office space, and from there, the data are uploaded to the Data Storage Server. Metadata are tracked using both Peregrine and the Digital Tool web interface. Powder bed imaging data are captured and analyzed in real-time by Peregrine. The raw imaging data and analysis results are uploaded automatically to the Data Storage Server.

\subsubsection{Data Visualization and Analysis}

The in-situ data produced by the ConceptLaser X-Line 2000R are analyzed by trained machine operators and data team members using the Peregrine software package. A total of four ConceptLaser X-Line 2000R builds were analyzed in FY20. Because this machine was nonoperational for the majority of 
CY20, only limited analysis results are available for FY20. However, results and capabilities are expected to be very similar to those reported for the ConceptLaser M2 machine. A broader overview of Peregrine is provided in the Digital Platform supplementary material.

\section{CONCLUSION}

This report proves that image-based defect detection coupled with time series data analysis in LPBF technology is a very reliable methodology for in-situ quality control. Multiple AI-based techniques have been developed to address these problems, and techniques have been successfully deployed on all powder bed systems being used by the TCR program and even other systems that are available at the MDF. Moving forward, this work will focus on (1) achieving higher resolution defect detection using a new 150 MP imaging sensor, and (2) processing new classes of defects specific to thin wall structures.

\section{REFERENCES}

[1] L. Scime, D. Siddel, S. Baird, and V. Paquit, "Layer-Wise Anomaly Detection and Classification for Powder Bed Additive Manufacturing Processes: A Machine-Agnostic Algorithm for Real-Time Pixel-Wise Semantic Segmentation," Addit. Manuf., p. 101453, Jul. 2020.

[2] L. Scime and J. Beuth, "Using Machine Learning to Identify In-Situ Melt Pool Signatures Indicative of Flaw Formation in a Laser Powder Bed Fusion Additive Manufacturing Process," Addit. Manuf., vol. 25, pp. 151-165, Jan. 2019.

[3] C. A. Steed, J. R. Goodall, J. Chae, and A. Trofimov, "CrossVis: A Visual Analytics System for Exploring Heterogeneous Multivariate Data with Applications to Materials and Climate Sciences," Graph. Vis. Comput., vol. 3, p. 200013, 2020. 


\section{APPENDIX A. TCR DIGITAL PLATFORM}

\section{A-1. THE DIGITAL PLATFORM}

The Digital Platform encompasses the design intent information, networking and computing infrastructure, in-situ sensing hardware, metrology and characterization processes, data storage and management systems, and software and analytics tools necessary to create and effectively leverage a Digital Thread (aka a digital twin) for each part and test specimen created for the TCR program. Components of the Digital Platform which have TCR-specific definitions are capitalized throughout this report for clarity. The backbone of the Digital Platform is a searchable Database linking data from component design, in-situ sensors, characterization, user-entries, machine calibration Timelines, and feedstock utilization Timelines. This information is physically stored on various Data Servers and is accessible via the Digital Tool web interface and associated application programming interfaces (APIs). The Digital Tool and APIs are hosted on the Database Server. Data are processed and visualized on Compute Servers or local user computers (e.g., desktops and laptops) using various ORNL-developed Software Tools; the Software Tools access the Database using standardized APIs. Figure A-1 provides an overview of the Digital Platform.

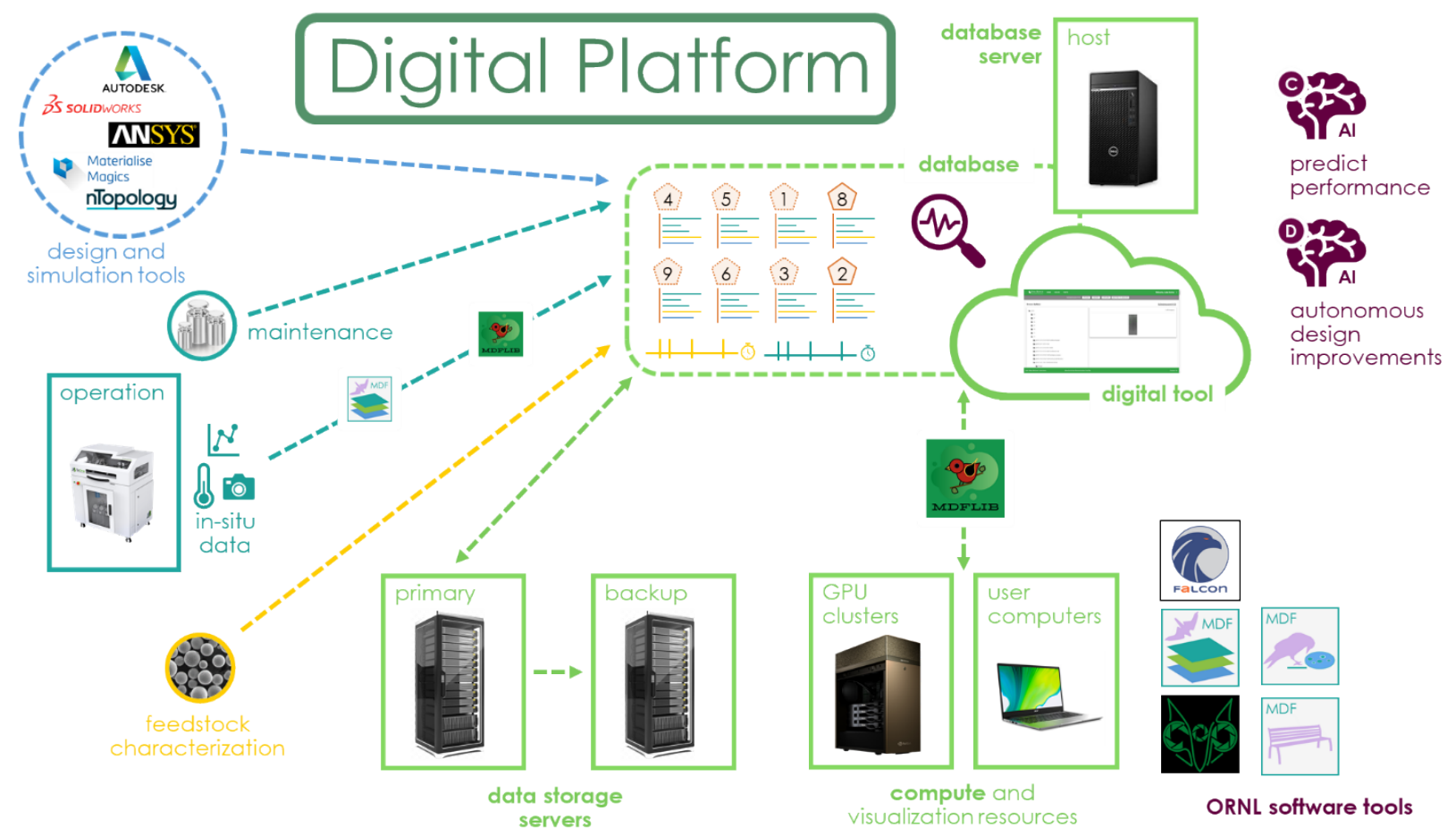

Figure A-1. An overview of the Digital Platform.

Each physical TCR component and test specimen will have a corresponding Digital Thread. Each Digital Thread is composed of building blocks referred to as Operations. Operations may be manufacturing processes, material characterization techniques, maintenance procedures, or purchases. Operations may act on a physical component, material feedstock (e.g., powder or wire), or a machine (e.g., a 3D printer). Example Operations include laser powder bed additive manufacturing, annealing, wire electrical discharge machining, chemical vapor infiltration, scanning electron microscopy, tensile testing, purchasing a component from a supplier, and laser calibration. Some Operations may also instantiate new 
parts or split an existing parent part into multiple child parts. The Digital Thread for a child part includes the thread for its parent.

Functionally, each Digital Thread is a list of Operations which have been performed on a given component and any corollary Operations that compose the machine maintenance and feedstock utilization Timelines. The Database stores these Digital Threads and allows users to access the data (e.g., in-situ powder bed images) associated with each Operation. A combination of the Digital Tool web interface and various Software Tools are used to visualize and analyze the Operation data. Data from multiple Operations may be spatially co-registered and visualized using certain Software Tools. Figure A-2 shows a representation of an example Digital Thread.

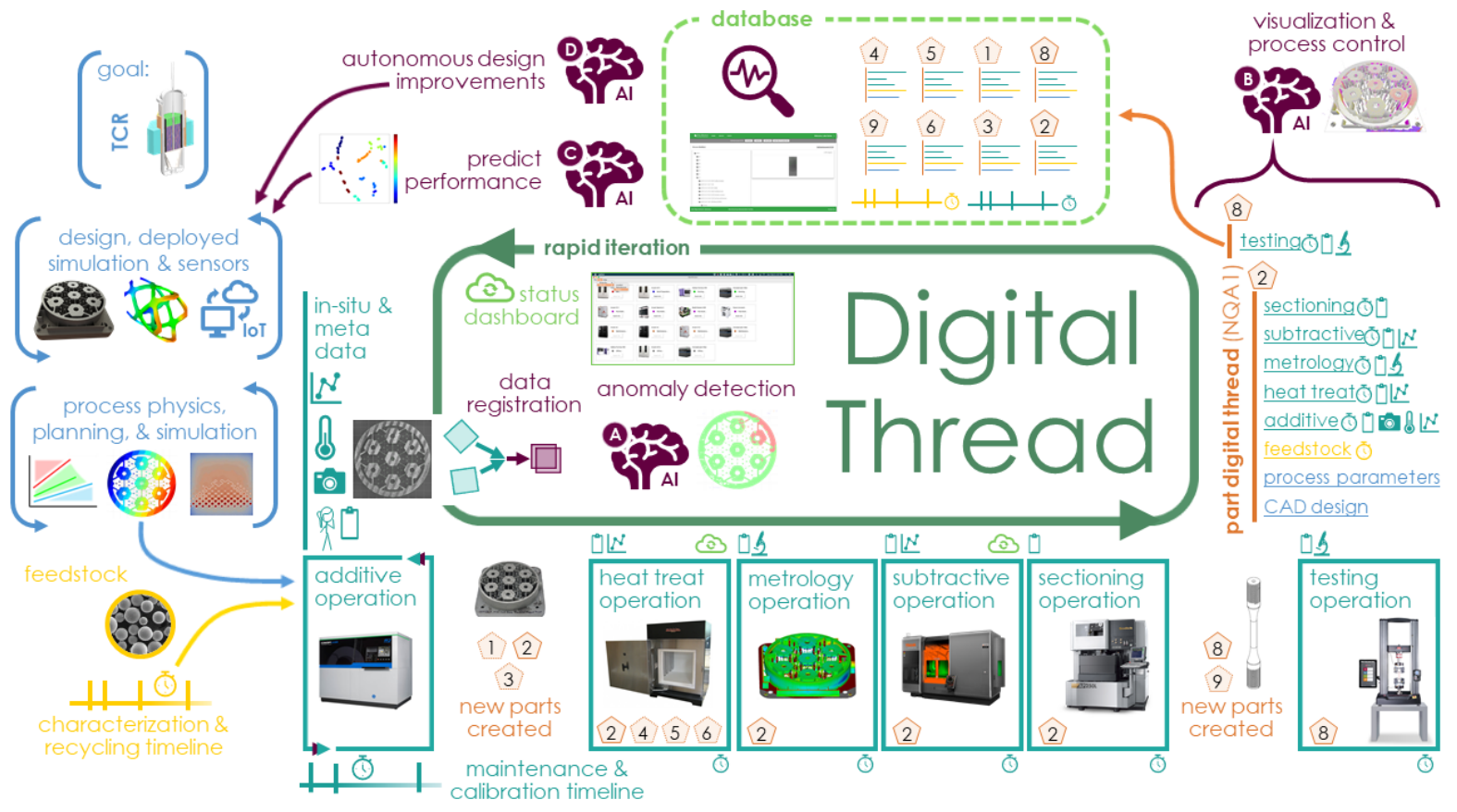

Figure A-2. A representation of an example Digital Thread. The process begins in the upper left-hand corner and follows the arrows counter-clockwise.

At a more granular level, information is incorporated into a Digital Thread as part of the Digital Workflow. Many different types of users may interact with a given Digital Workflow, including reactor designers, advanced manufacturing engineers, machine technicians and operators, material scientists, data analysts, program managers, and public communications specialists. The composition of each Digital Thread, and therefore the corresponding Digital Workflow, varies dramatically, depending on the particular component. As an example, Figure A-3 shows a simplified Digital Workflow for a binder jetprinted silicon carbide reactor fuel element. 


\section{Digital Workflow}

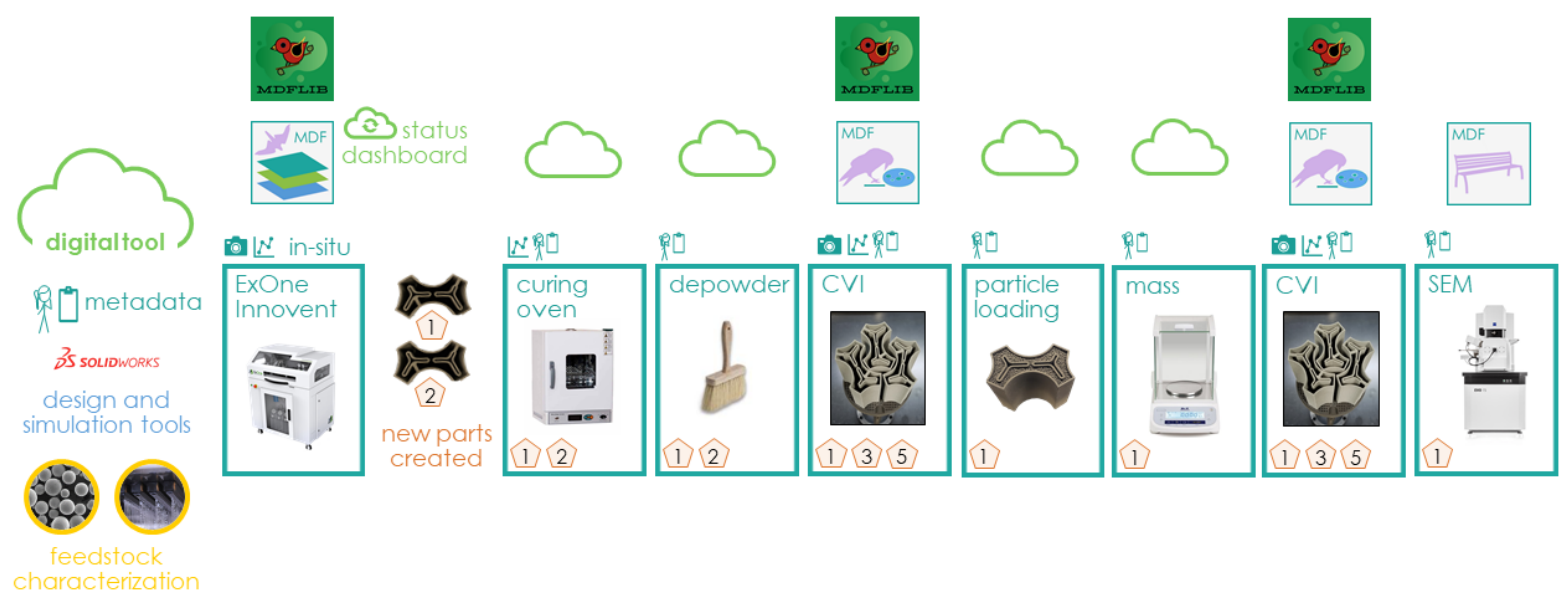

Figure A-3. A simplified Digital Workflow for the manufacture of a silicon carbide fuel element.

A primary goal of the TCR program is the prediction of reactor-relevant part properties based on in-situ data collected during various advanced manufacturing processes. To this end, augmented intelligence (AI) algorithms will ingest collections of Digital Threads in order to identify correlations between in-situ data and part properties. We deliberately refer to such algorithms as augmented intelligence instead of artificial intelligence to convey the necessity of keeping expert humans and their physics-based understanding of the world "in the loop" throughout these analyses. Critically, part property prediction will be accomplished through a relay of AI algorithms, with each hand-off leveraging human expertise to inspect the AI performance and to point the subsequent AI in the correct direction. This relay approach is essential to ingest the highly unstructured and extremely high dimensional in-situ sensing data and to successfully link it to part property measurements without requiring a prohibitively large number of exsitu characterization experiments. To further clarify the terminology used throughout the TCR reports, $A I$ is considered to be a broad umbrella of algorithms which includes heuristics, machine learning (ML), and deep learning (DL). The in-situ/ex-situ property correlation efforts for FY20 are described in a separate TCR report titled Viability of Data Analytics to Ascertain Component Performance for Additive Manufacturing. Consistent tracking of each reactor part and test specimen is a critical aspect of this approach. Development of a robust, integrated, and automatic specimen naming scheme was completed in FY20 and is illustrated in Figure A-4. 


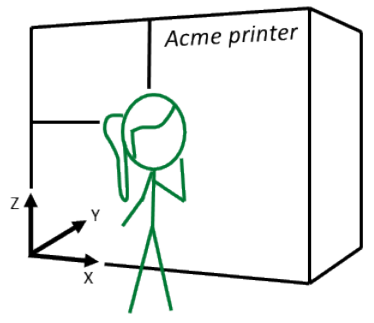

ISO/ASTM 52900:2015(E)
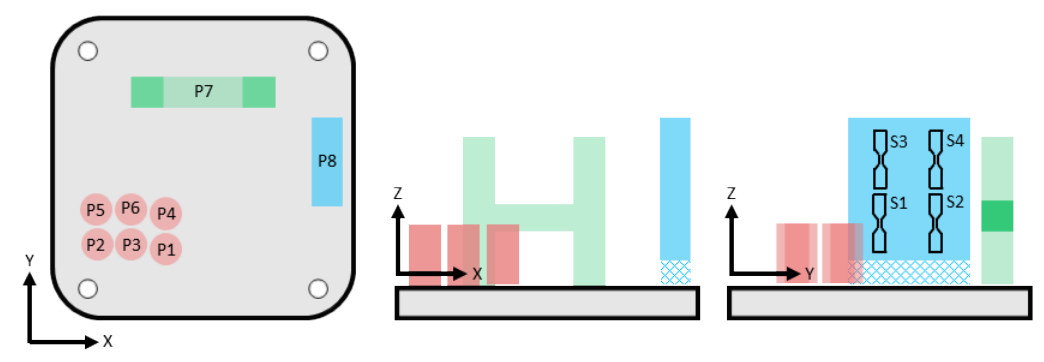

Numbering Order:

Specimen Types:

1. Search $+Z$

- "Part" geometry (P)

2. Search $+Y$

- "Sample" geometry (S)

3. Search $+X$

Figure A-4. Specimen numbering scheme following the ASTM 52900:2015(E) standard [1].

Importantly, AI is used for data analysis and visualization throughout the Digital Thread. AI usage for the TCR program can be approximately separated into four different application areas: descriptive, diagnostic, predictive, and prescriptive. Descriptive AI is used to analyze raw data, an example being segmentation of powder bed images and identification of process anomalies. Diagnostic AI summarizes large datasets and presents them to a human user or another algorithm. A diagnostic AI might flag certain layers of an additive manufacturing Operation for closer inspection by a human, or it might decide on an autonomous process intervention to attempt to correct a detected defect. A predictive AI seeks to predict part properties (e.g., fracture toughness) based on in-situ data, process parameter information, and part geometry. Demonstration of a predictive AI is an end goal of the TCR program. Finally, a prescriptive AI might would autonomously modify a part design in order to improve the predicted performance; this class of AI is beyond the scope of the TCR program, but it is the ultimate goal for the Digital Platform.

In FY20, the Digital Platform transitioned from a conceptualization framework and a set of stand-alone tools into a highly flexible, scalable architecture with increasing integration between the Software Tools and the Digital Tool. The physical infrastructure needed to support the Digital Platform includes (1) a optical fiber-based dedicated network to connect all machines to the facility digital backbone,

(2) dedicated Wi-Fi networks for Internet of Things (IoT) sensors, (3) redundant storage systems, and

(4) multi-GPU (graphics processing unit) compute systems for AI model training and AI at the edge.

FY20 also saw the standardization of the onboarding process for new Operations to be recognized by the

Database. The remaining sections of this supplementary appendix describe the various components of the Digital Platform, including the ORNL-developed Software Tools. Changes and improvements made in FY20 are highlighted. 


\section{A-2. DATA STORAGE INFRASTRUCTURE AND DATABASE ARCHITECTURE}

There is a diverse variety of data produced by a Digital Workflow during different Operations. The data produced varies with respect to the data source, data type, data format, and the scale of the data volume. The standardization of this wide variety of complex data is a challenging problem to solve. To address this complex data organization, the data are structured, organized, and stored on a dedicated Storage Server. This standardization facilitates users and services with improved data search, management, access, and retrieval functionality. The data are organized by the data source (i.e., Operation) and are further organized by the date of the Operation. The dedicated data Storage Server is used as the primary location for storing and accessing the data, whereas a secondary data Storage Server is used for monthly data backups. Some of the benefits which are achieved using a dedicated Storage Server for TCR data include:

1. The Data Storage Server acts as a centralized data repository, and the data are available to all the computers within the same network.

2. Multiple users can access the same dataset, eliminating the need to make duplicate copies of data for different users.

3. Data integrity is maintained because the latest datasets are centralized and accessible.

4. The server provides better data management and implementation of security protocols, and it allows data access protocols to ensure that TCR data can only be accessed by authorized users.

A Database Server was installed, and a relational database was developed to store and organize the metadata produced from Operations and associated with the Digital Threads. PostgreSQL was selected as the standard database protocol; PostgreSQL is an open-source, object-relational database system. It is one of the most widely used database systems because of its strong community support, scalability, reliability, and consistent performance. PostgreSQL also provides a robust set of features to support complicated Digital Workflows.

The purpose of the PostgreSQL Database is to facilitate metadata collection, data organization, and storage, along with search and retrieval on the datasets stored on the Data Storage Server. To achieve these requirements, the Database was designed so that relevant data are stored in individual schemas. Some of the schemas present in the current database are as follows:

1. Users: contains information about authorized users of the Database

2. Roles: contains information about which users have access to each Operation and dataset

3. Projects: contains information about different projects at the MDF

4. Machines: contains information about different manufacturing equipment

5. Materials: contains information about the feedstock materials and their manufacturers

6. Operations: contains information about Operations supported by the Digital Workflows

7. Parts: contains information about all manufactured specimens and the Operations which compose their associated Digital Threads.

In addition to the schemas listed above, there are additional AM machine-specific schemas to store information about build datasets and the files in each dataset. These schemas contain metadata which populate the Digital Thread of each manufactured component.

\section{A-3. DIGITAL TOOL (WEB INTERFACE AND APIS)}

The Digital Tool is a web-based application developed in Vue.js at ORNL. Vue.js was selected for the development of this platform because of its small size, high performance, and excellent flexibility. Vue.js also has detailed documentation available, and it supports microservices architectures for developing web applications. Microservices architectures support incremental and modular growth of the application, thus 
making it a suitable framework for developing scalable infrastructure. Figure A-5 illustrates a microservices architecture.

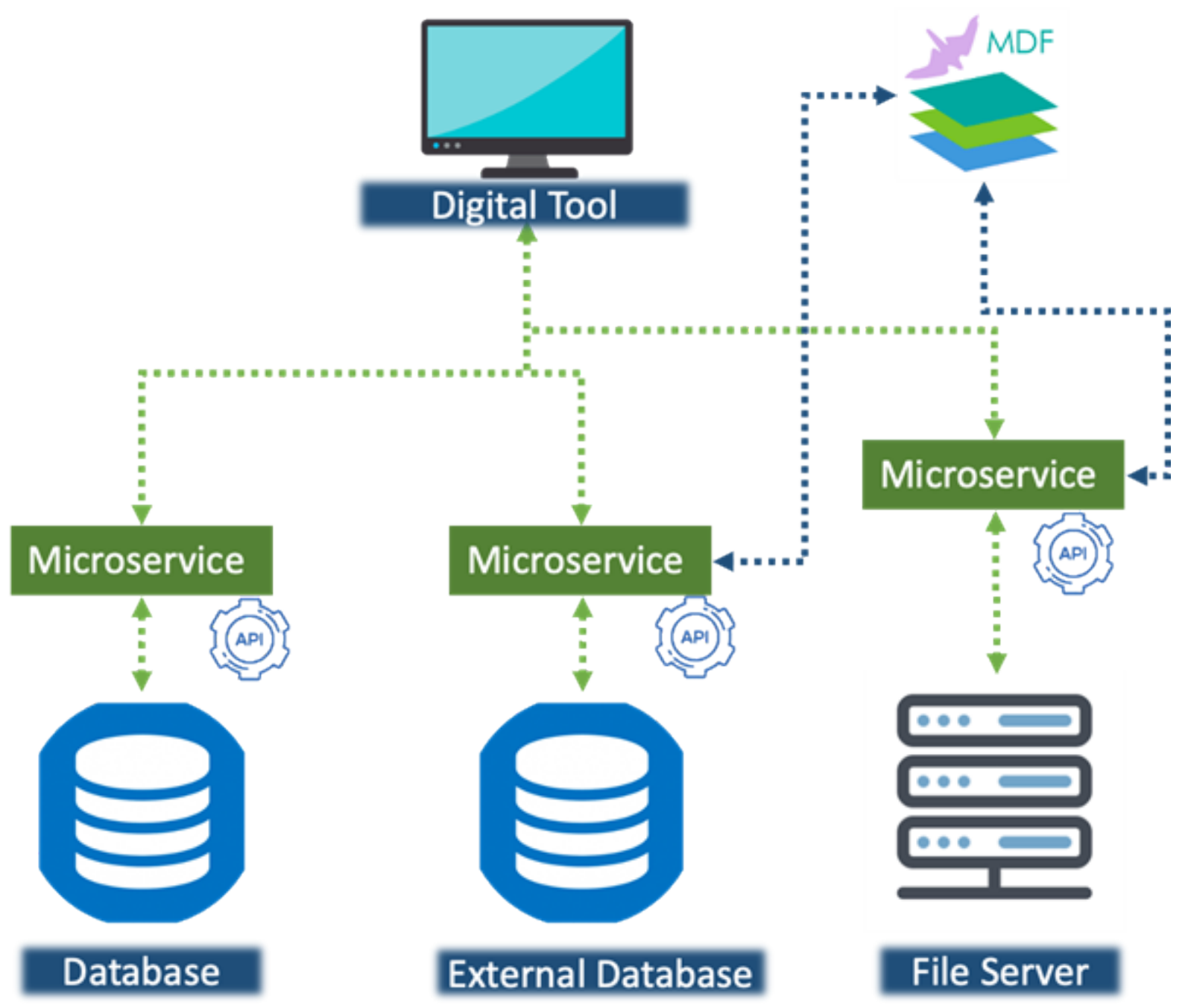

Figure A-5. Representation of the microservices-based architecture utilized by the Digital Tool.

The main components of the Digital Tool microservices architecture are as follows:

1. Digital Tool: the web application which is developed using JavaScript and html

2. Microservices: RESTful services developed in Python flask. These services run independently as a service and can communicate and pass information between data sources and the Digital Tool. Additionally, these services can be utilized by various Software Tools developed by the TCR data team. Currently, there are multiple independent microservices running which vary in functionality from querying databases, updating databases, and performing data transfer actions.

3. Data sources: microservices act as an interface between the applications and data sources. In this architecture, the data sources are the PostgreSQL Database and central Data Storage Server.

The Digital Tool provides online forms (Figure A-6) to help technicians record metadata before, during, or after an Operation. These metadata tracking forms were developed based on feedback from technicians 
and the ultimate data users. The forms are validated to ensure that the metadata collected are standardized, complete, and correct.

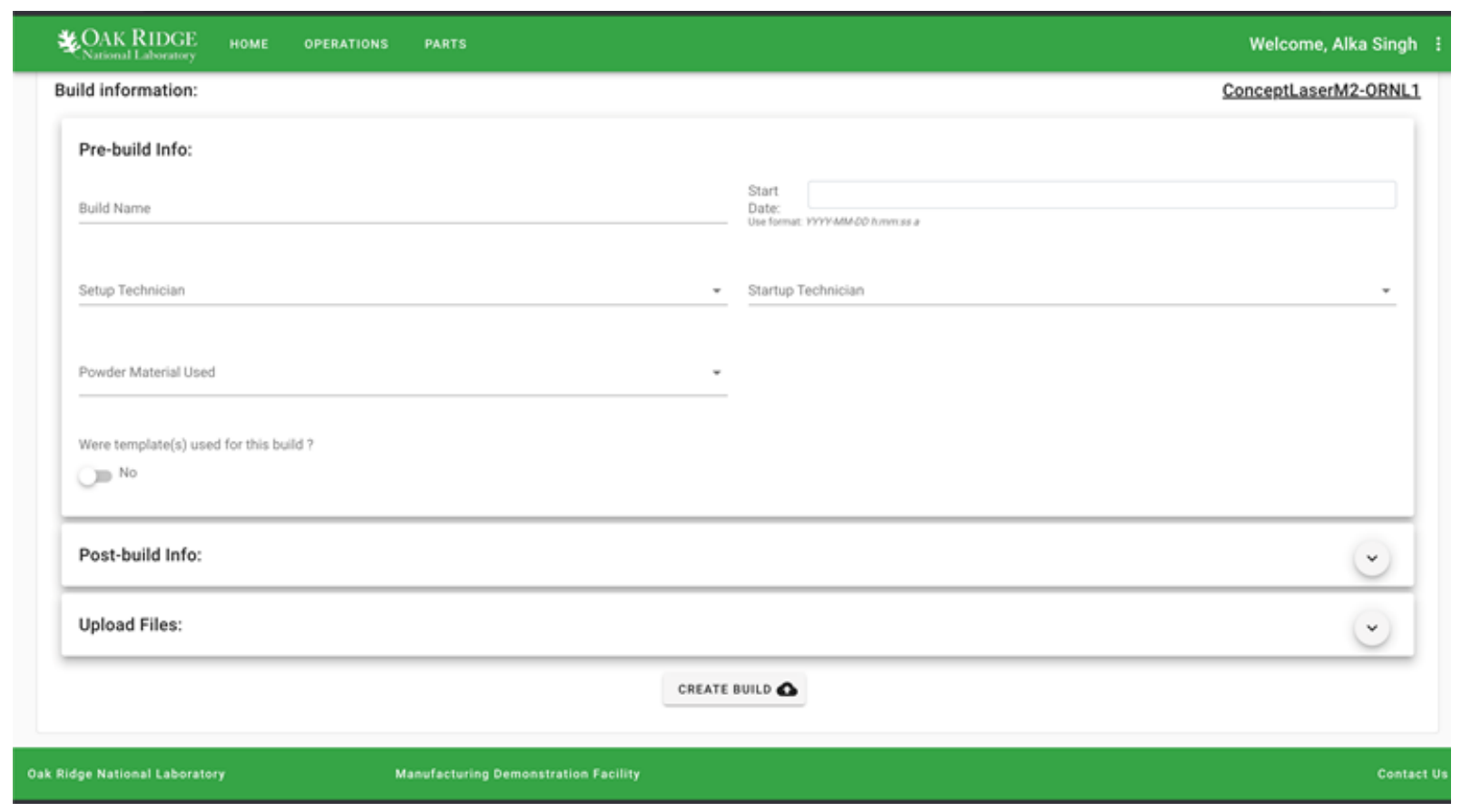

Figure A-6. An example metadata entry form for an Operation.

The Digital Tool allows users to search (Figure A-7) the existing datasets based on the recorded metadata. This feature enables users to narrow down the desired datasets based on specific metadata, as well as less structured criteria such as the processing parameters used for an additive build. 


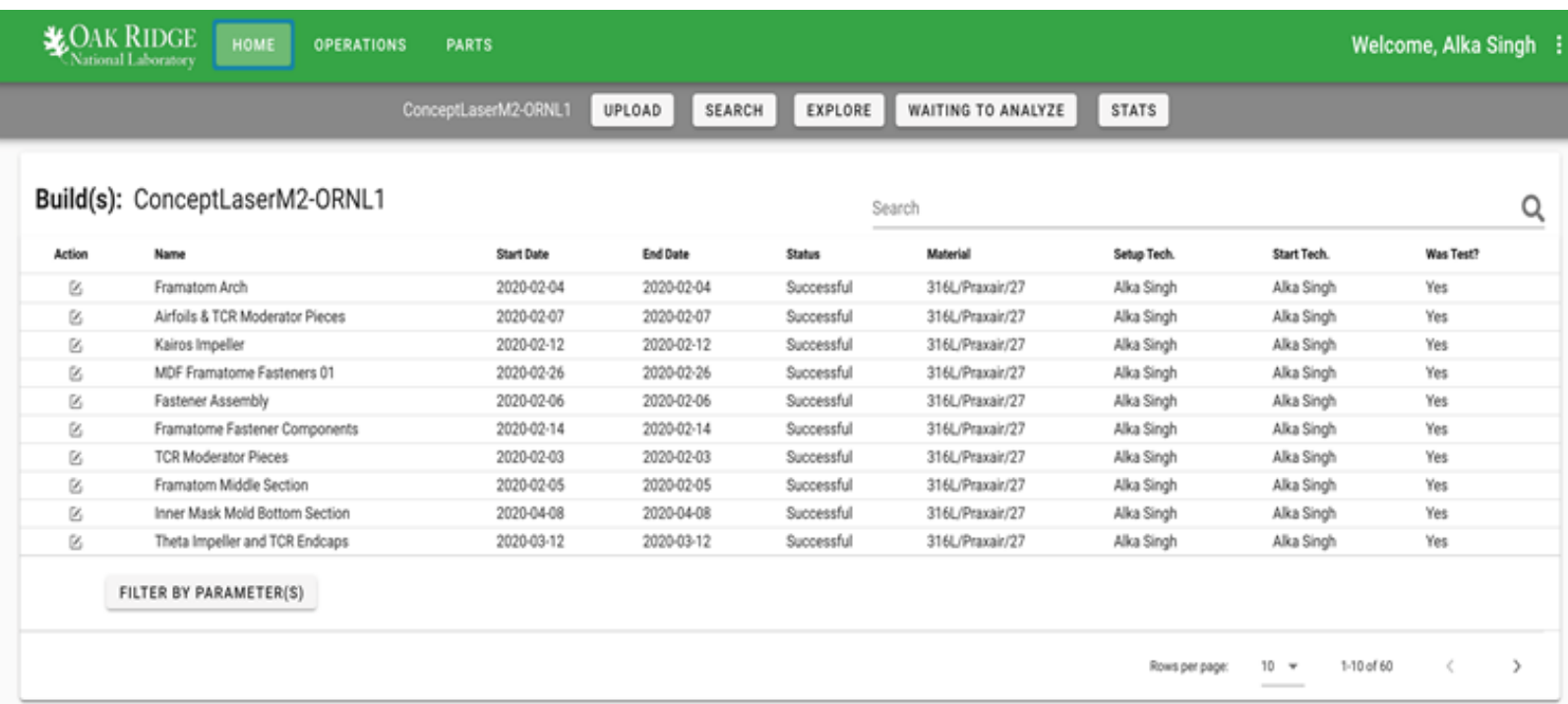

Figure A-7. An example outcome of a search action.

The Digital Tool also allows users to explore the existing additive manufacturing build datasets contained on the Data Storage Server. There are multiple data viewers (Figure A-8) to support exploring different data types including image viewers, STL viewers, PDF viewers, and log file viewers. This feature allows multiple users to access data simultaneously without requiring direct access to the stored data, thereby preventing accidental data modification.

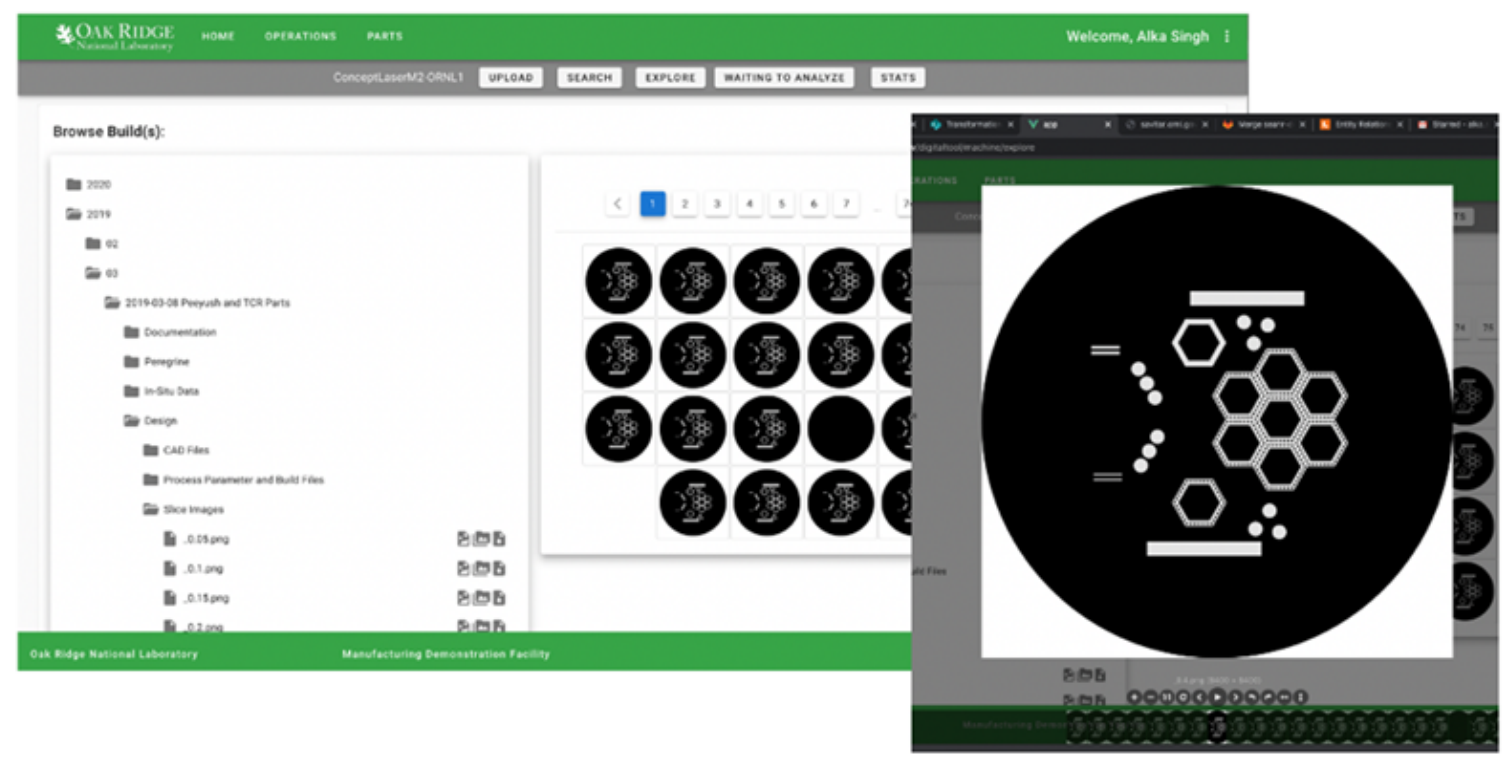

Figure A-8. An example of the image viewer feature. 


\section{A-4. PEREGRINE}

Peregrine is a Software Tool developed in Python at ORNL. Peregrine is intended for use with powder bed AM machines (i.e., printers), including LPBF, electron beam powder bed fusion EB-PBF, and binder jetting technologies. In its fullest implementation, Peregrine autonomously collects and analyzes layerwise imaging data, provides remote monitoring and process intervention capabilities, tracks metadata and part information, produces advanced visualizations of both the underlying data and the AI analysis results, and enables identification of correlations between in-situ data and process parameters or ex-situ measurements.

Peregrine's core capability is pixel-wise anomaly detection based on layer-wise images of the powder bed. This semantic segmentation of the data is achieved using a novel, deep learning algorithm referred to as a dynamic segmentation convolutional neural network (DSCNN). The DSCNN architecture was designed specifically to overcome many of the challenges common to powder bed imaging data and is fully described in Scime et al. [2]. Beyond this core capability, Peregrine also empowers users with several advanced analytics and data tracking tools.

1. Each layer in a build can be flagged based on the DSCNN pixel segmentation results using either a set of user-defined heuristics (i.e. rules) or a learned ML model, referred to as the DSCNNPerceptron (DSCNN-P).

2. Peregrine provides advanced data visualization, allowing the user to visualize the raw data, DSCNN, and DSCNN-P results in various formats. These visualizations include image overlays, plots of time series data, frequency analyses, specimen quality scoring, data projections in each orthogonal plane, 3D reconstructions, and timelapse videos of the build.

3. Peregrine provides automatic specimen numbering and statistics. Up to two sets of CAD geometry information can be associated with each build, including the as-built geometries, which are referred to as parts, and the geometries of any samples removed from the components after the build is complete, such as a tensile bar. The term specimen is used by Peregrine to refer to either a part or a sample.

4. Metadata can be tracked for each build, along with reference images, log files (supported printers only), coaxial time series data (supported printers only), and registered ex-situ data. Peregrine also includes 2.5D registration capability, allowing users to overlay ex-situ data such as x-ray computed tomography (XCT) slice images on top of in-situ data and DSCNN results.

5. Peregrine is capable of direct data collection and real-time analysis using most USB and ethernet cameras. During a live analysis, Peregrine can send alert emails to technicians, execute customizable macros to interface with a printer's user interface to effect process interventions, and provide remote viewing of the build status via a companion instance of Peregrine.

6. A limited number of statistics tools are also provided to enable the user to investigate potential correlations between Peregrine's in-situ results and process parameters or ex-situ measurements.

A high-level overview of Peregrine's capabilities is presented in Figure A-9, and Peregrine's internal data flow is summarized in Figure A-10. The figures illustrate bundling of all of the layer-wise imaging data into a single calibrated image stack, followed by a tiling operation before the data are fed into the DSCNN. The pixel classifications predicted by the DSCNN are then compared to the template image, and potentially the raw powder bed imaging data, using pixel heuristics. These comparisons create the final pixel classifications observed by the user. The output of the DSCNN is fed into the DSCNN-P to allow for labeling of the entire build layer, or image stack. Finally, the labels assigned by the DSCNN-P are combined with the global heuristics and presented to the user as layer flags. 


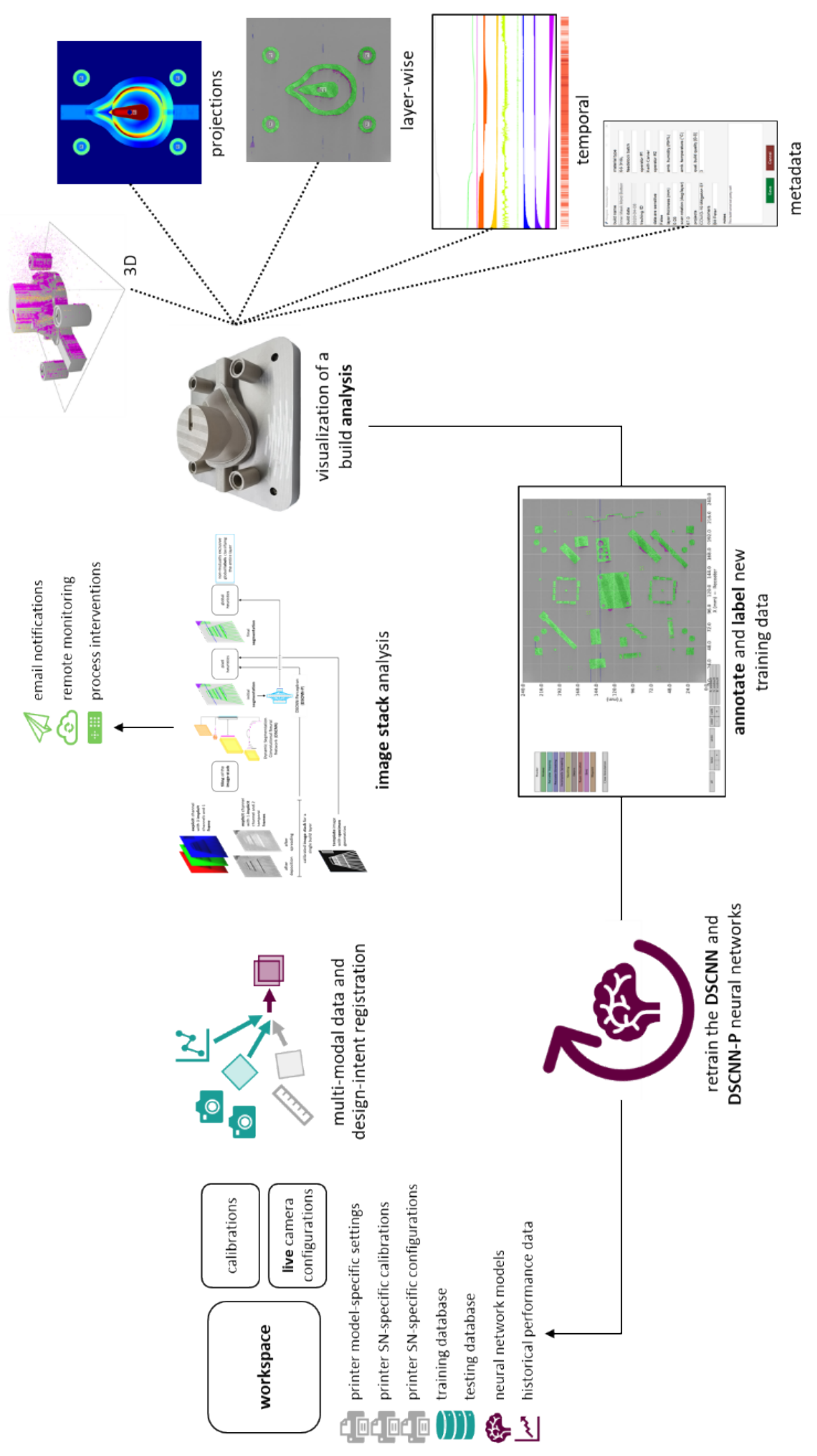

Figure A-9. Overview of Peregrine's capabilities and operation. 


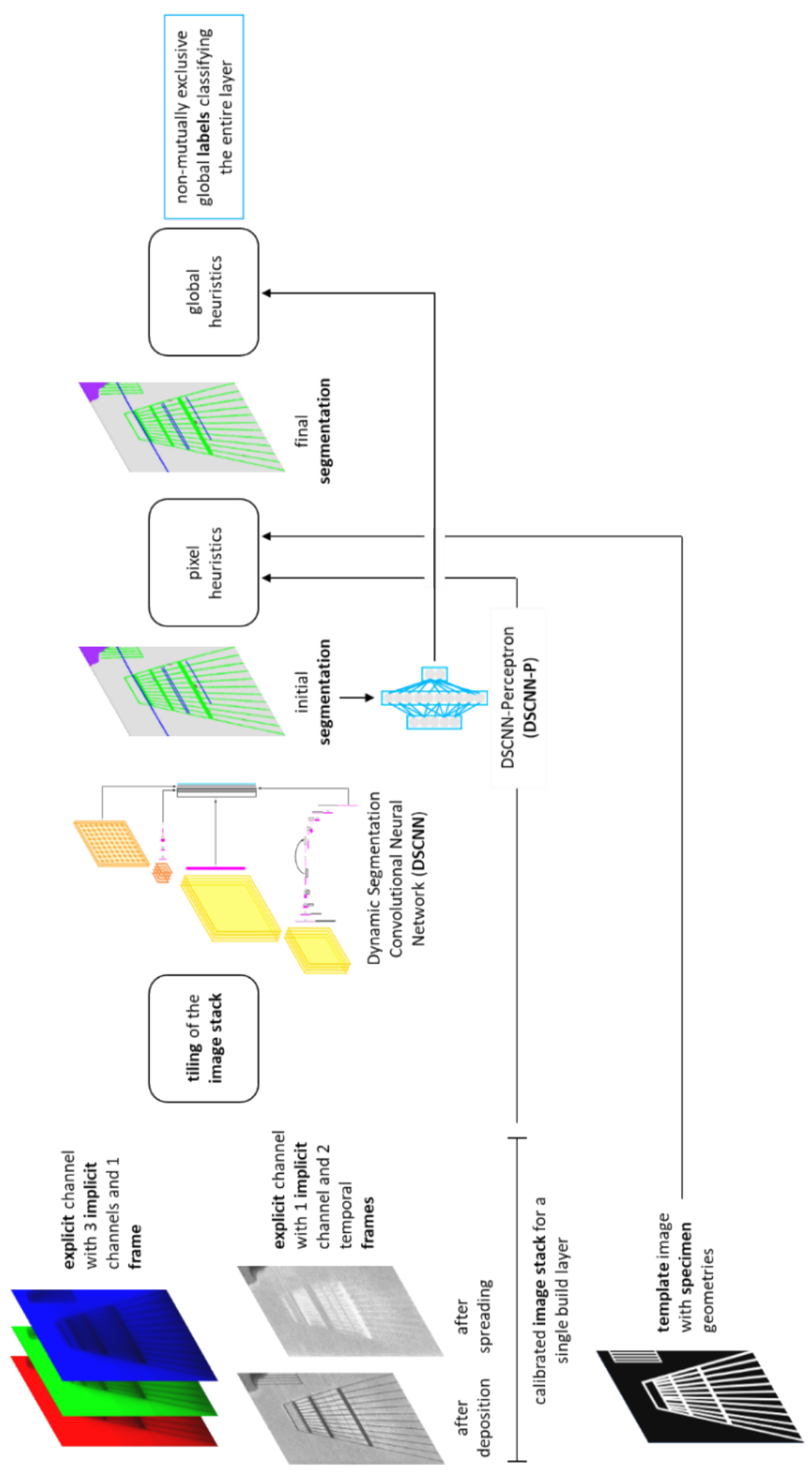

Figure A-10. Peregrine's internal data flow. 
Peregrine has been under development since FY18. It is currently on major version 20 and is used by approximately ten members of the TCR team. Users include data scientists, material scientists, and machine technicians. Over 100 pages of documentation and training materials have been written for Peregrine in FY20. In FY20, ORNL authored a journal paper describing the DSCNN which was published in Additive Manufacturing. Peregrine can now be licensed to companies, with over ten companies already expressing interest. Below is a list of the major Peregrine improvements completed in FY20.

1. The software release process was formalized, including documentation, versioning, archiving, and bug reporting.

2. An immutable change log is now created for each build analysis to record all data edits and allows builds can now be marked as containing sensitive data.

3. Peregrine has been integrated into the Digital Platform, allowing data to be passed to and from the Database. Printer settings, calibrations, trained neural networks, and analyzed builds are hosted on the Data Storage Servers and are transparently accessible to all users at ORNL and working remotely. Any trained user can directly update training data to improve AI model performance. Versioning improvements allow changes to be tracked as multiple users modify shared files.

4. A part numbering standard was introduced for the TCR program. Peregrine automatically numbers all specimens for each build. Automatic and consistent numbering is absolutely critical for tracking and maintaining the hundreds of thousands of Digital Threads to be stored on the Digital Platform.

5. The DSCNN architecture was upgraded to DSCNNv2. This architecture improves accuracy, segmentation of curvilinear structures, and segmentation of smaller structures, and it also reduces inference time and tiling artifacts. DSCNNv2 dramatically improves training flexibility and follows more industry standards for neural network design.

6. The entire data loading architecture was rewritten to allow for color images, 16-bit images, a truly arbitrary number of cameras, an arbitrary number of temporal frames, and more complex interlayer lighting differences. These changes make Peregrine much more flexible as the imaging systems on several of the printers are improved in FY21.

7. XCT data can now be co-registered to the in-situ imaging data and overlaid on top of the DSCNN predictions within Peregrine.

8. Data collected live are fully analyzed and accessible in real-time - this is critical for handling the massive TCR data burden.

9. The DSCNN-Perceptron can now be used to flag layers to bring them to the attention of a user or machine operator, and early results using this capability are promising. A training data collection and labeling campaign began in FY20 and will continue in FY21 to improve the utility of the DSCNN-Perceptron predictions.

10. The entire user interface was rebuilt based on over a year of user feedback. The primary goal was to enable faster, more efficient review of the analyzed build data and to enable crowdsourcing for collection of new training data.

11. The ConceptLaser X-Line 2000R machine type is now recognized by Peregrine.

12. Several artifacts can now be removed from the QM Meltpool data.

13. Log files can now be parsed for the ExOne Innovent and ExOne M-Flex machines.

\section{A-5. RAVEN}

Raven is a Software Tool developed in Python at ORNL. Raven is intended for use with the chemical vapor infiltration (CVI) process. Specifically, it allows the user to track the locations of the binder jet 
printed parts prior to insertion into the CVI furnace. CVI technicians will take a series of images of the parts as they are loaded onto the furnace platforms, or "grid plates." These images are taken under controlled lighting conditions and background subtraction, along with other classical computer vision techniques. The images are used to determine the boundaries of each part. Each part is then assigned an identifier number produced by the Peregrine Software Tool during the instantiating printing process. The part locations and their corresponding identifiers are saved to a file which is uploaded to the Database via the Digital Tool web interface.

Development of Raven began in FY20, and it is currently on major version 1. Raven has not yet been deployed to users because COVID-19 restrictions delayed assembly and testing of the physical imaging system. Bringing Raven fully online is a priority for early FY21. Approximately 10 pages of documentation and training materials were written for Raven in FY20. The intended users of Raven will be the CVI machine technicians.

\section{A-6. SCOPS}

SCOPS is a set of imaging systems and an analysis method developed to analyze the complex thermomechanical history of parts printed with directed energy deposition (DED). The system uses stereo digital image correlation (DIC) to 3D map surface roughness features on parts as they are printed and tracks their motion over time. This allows for direct, in-situ, noninvasive deformation and strain measurements over the entire part. This is combined with infrared thermal imaging to measure material expansion and contraction as a function of heat flow through the part. The SCOPS system is summarized graphically in Figure A-11.

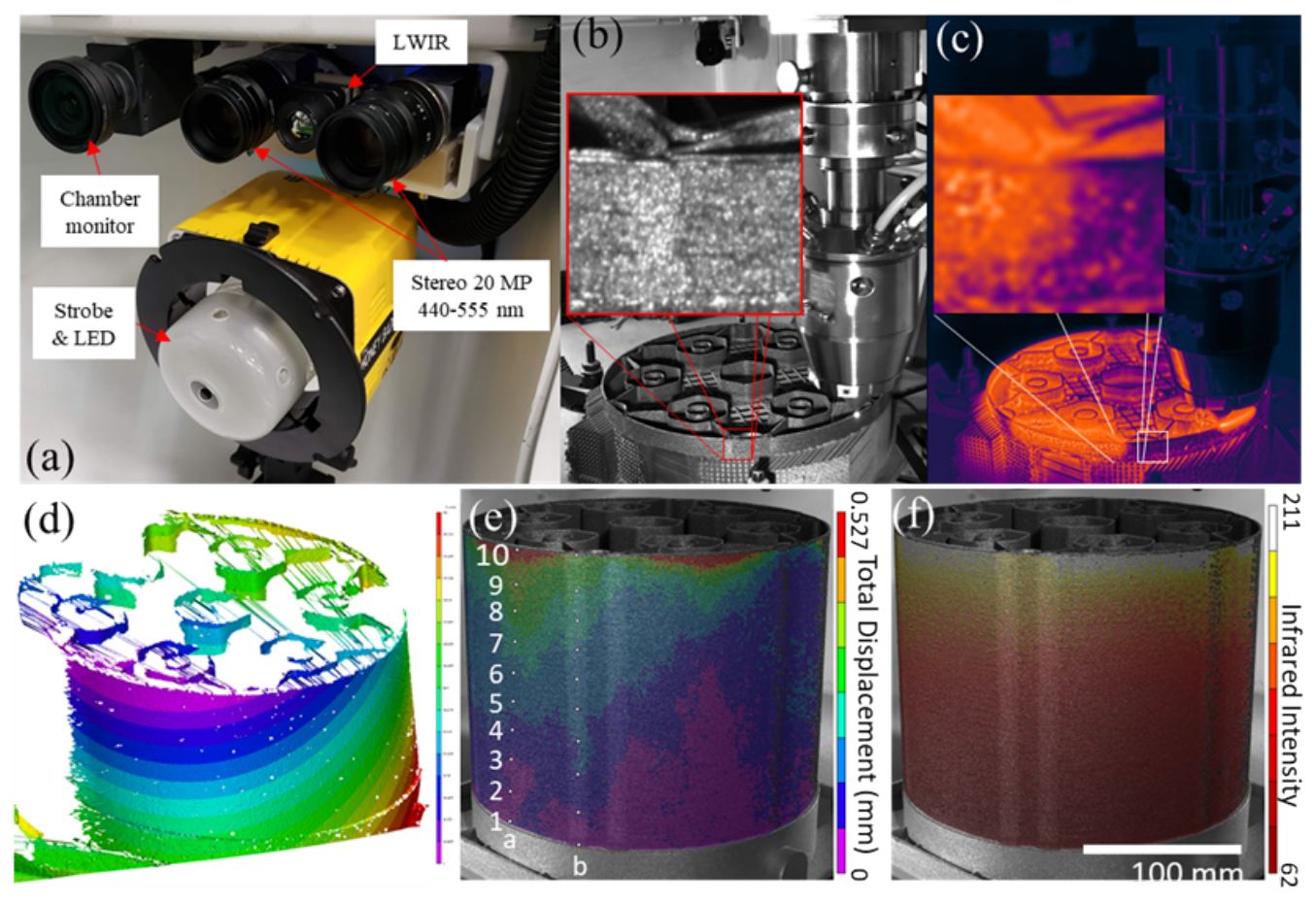

Figure A-11. (a) SCOPS imaging system, two stereo high-resolution visible cameras and an infrared camera, (b) visible trackable surface roughness of representative deposition, (c) infrared imaging showing thermal dissipation, (d) 3D map constructed from surface features, (e) tracked deformation induced from a combination of residual strain and thermal expansion from (f) 3D mapped infrared signal showing hot regions. 
In FY20, two DED machines were outfitted with SCOPS: the BeAM modulo 400 and GKN cell 1. System hardware configuration was developed and optimized, as along with supporting software for automated video capture and archival. Blackbody calibration was performed, and thermocouple validation of infrared imaging was automated. A validation experiment comparing DIC to neutron diffraction residual stress measurements was performed. Information from SCOPS provides an in-situ measure of a previously inaccessible information stream, and it enables direct validation of finite element analysis (FEA) models, enhancing the accuracy of residual stress and microstructure simulation.

\section{A-7. PIGEON}

Pigeon was developed at ORNL and is both a Software Tool and a Python API. Pigeon is primarily an image segmentation software tool powered by a DSCNN. For more information regarding the DSCNN, users are referred to Scime et al. [2]. Pigeon's data flow is essentially identical to that represented in

Error! Reference source not found. for Peregrine. Some of Pigeon's key features include:

1. Pigeon accepts an arbitrary number of input channels, working seamlessly with both RGB and single channel images. Pigeon also supports an arbitrary number of temporal frames associated with each explicit channel. For example, if two spatially registered color images, each with two temporal frames, are provided, then there are two explicit channels and twelve implicit channels.

2. Pigeon accepts images of arbitrary bit depth (up to 16 bits).

3. Pigeon accepts images of arbitrary resolution while ensuring that the output segmentation is always at the resolution of the input image.

4. The DSCNN is designed to work well with very high-resolution images of $20 \mathrm{MP}$ or greater, even with limited GPU RAM available. It also provides sublinear analysis times, so doubling the image resolution will not double the inference time.

5. A low-resolution leg of the DSCNN always has a receptive field equal in size to the entire input image. This helps the DSCNN to learn to be robust to lighting variations and global scene changes.

6. The medium resolution leg of the DSCNN has a receptive field which can be dynamically configured by the user. This size of this tile should be based on domain knowledge such that it includes the appropriate amount of contextual information.

7. The DSCNN can learn prior probability distributions that are a function of pixel location within the global image.

8. Class-wise imbalances are not a significant issue, because automatic rescaling of the loss function ensures that the DSCNN learns even extremely rare classes.

9. Full transfer learning from any DSCNN to any other DSCNN is supported.

10. The user can create multiple workspaces for working on different problems.

11. The DSCNN also outputs a deep feature vector which can be used by a perceptron to classify the entire image stack.

Development of Pigeon started in FY20. It is currently on major version 1 and is used by several members of the TCR team. Over 30 pages of documentation and training materials have been written for Pigeon in FY20. Users include data and material scientists. Pigeon is intended primarily as a development platform for creating different characterization tools. For example, Pigeon can serve as the framework for multiple Software Tools, such as one dedicated to characterizing powder feedstock and another focused on analyzing AM microstructures. Efforts are already underway to use Pigeon for a number of different aspects of the TCR Digital Platform, with a primary focus on automated material characterization. 


\section{A-8. SIMURGH}

$\mathrm{XCT}$ is a process in which a 3D volume of an object of interest that represents attenuation coefficients of its comprising materials is reconstructed from 2D projections of the object acquired at different angles (views). XCT plays a critical role in nondestructive evaluation (NDE) and therefore certification/qualification of metal AM components. The quality of the 3D reconstruction algorithms used for dense metallic parts in complex systems and structures can be compromised by noise and artifacts such as streaks associated with metals, and a confounding effect called beam hardening (BH). Such artifacts and noise complicate the process of detection of salient defects (e.g., pores and cracks) in XCT images.

ORNL has developed an AI-based technique, Simurgh, that leverages CAD models of the parts, along with physics-based modeling, that shows significant improvement in XCT resolution by surpassing the state-of-the-art in suppressing BH, metal artifacts, and noise in preliminary tests on both synthetic and real data. Figure A-12 shows a block diagram of the Simurgh technique. Figure A-13 and Figure A-14 show example results on both synthetic and real data sets, respectively. For development and testing of the method, the data currently available at the MDF were used: XCT scans of printed airplane engine turbine blades. In FY21, these methods will be evaluated and developed further for application to TCRrelevant geometries.

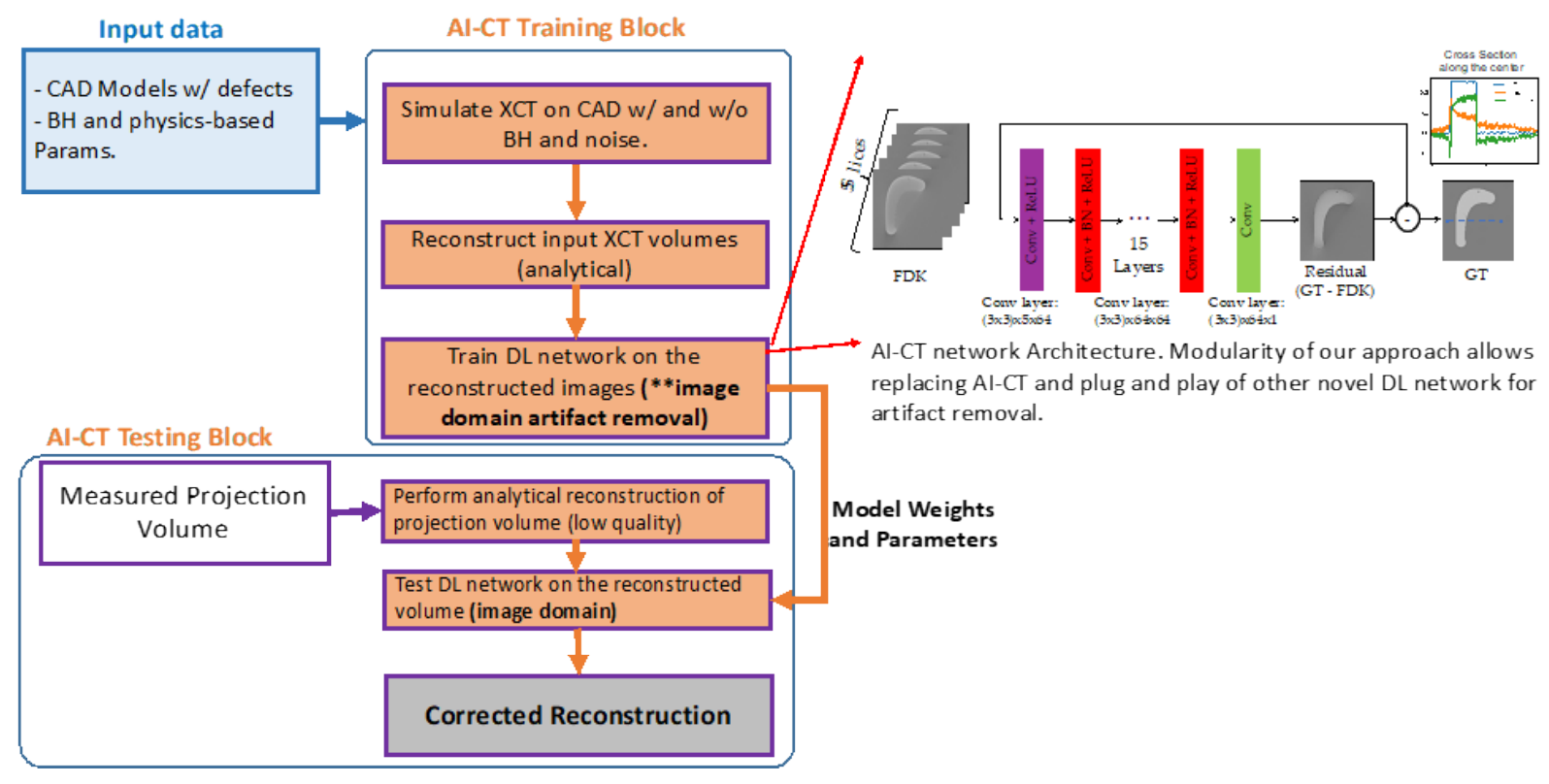

Figure A-12. Block diagram of the Simurgh framework. ORNL trains a convolutional neural network (AI-CT, top right) on the simulated (synthetic) data developed using CAD models and physics-based parameters. 


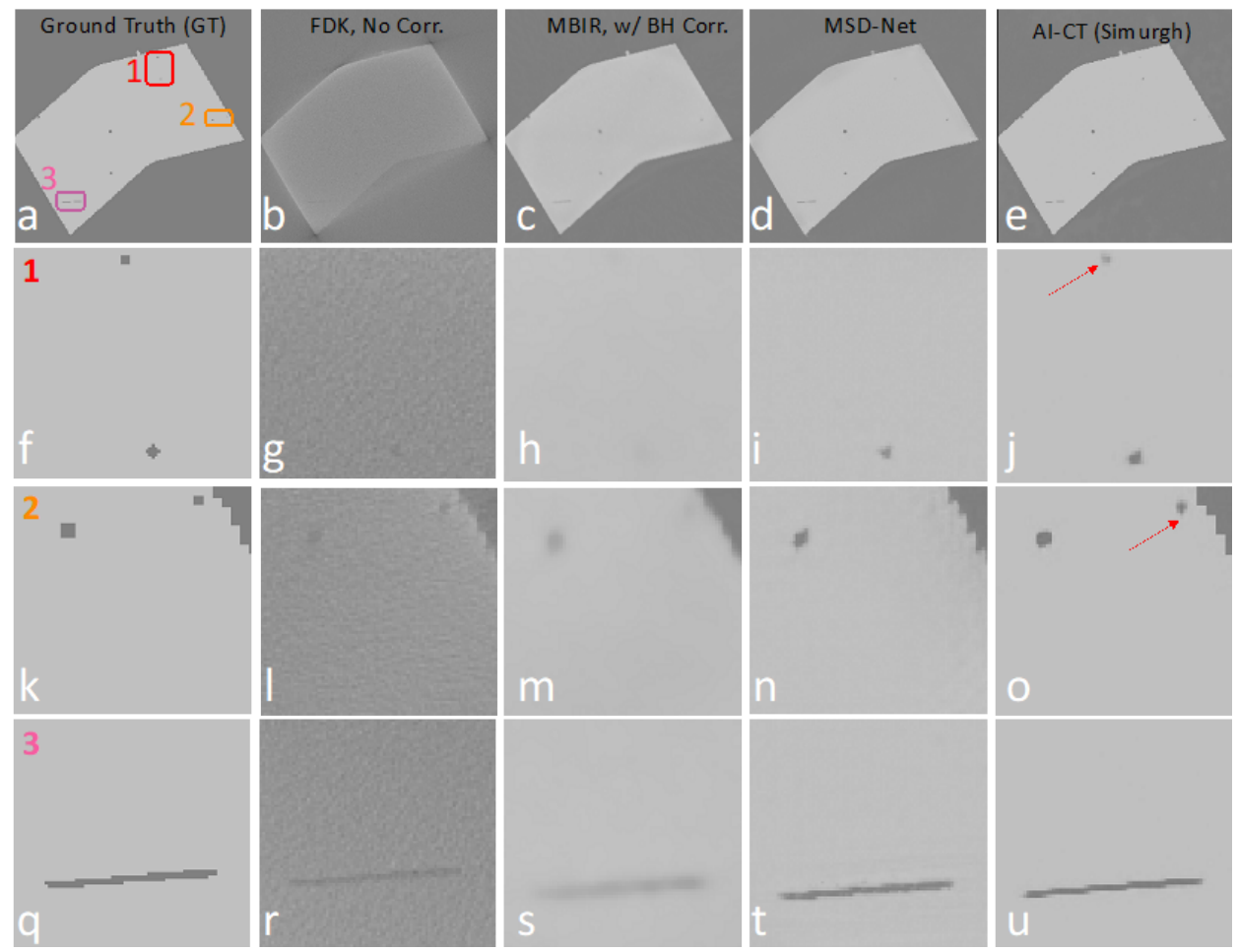

Figure A-13. Example test results on a synthetic data set. Two different neural networks (MSD-Net [3] and AI-CT [4], [5]) to emphasize the modularity of the approach. The method is also compared against uncorrected standard analytical (FDK) approach [6] and state-of-the-art model-based iterative reconstruction (MBIR) [7]. Several areas of interest are highlighted to demonstrate the superiority of the work against the state-of-the-art.

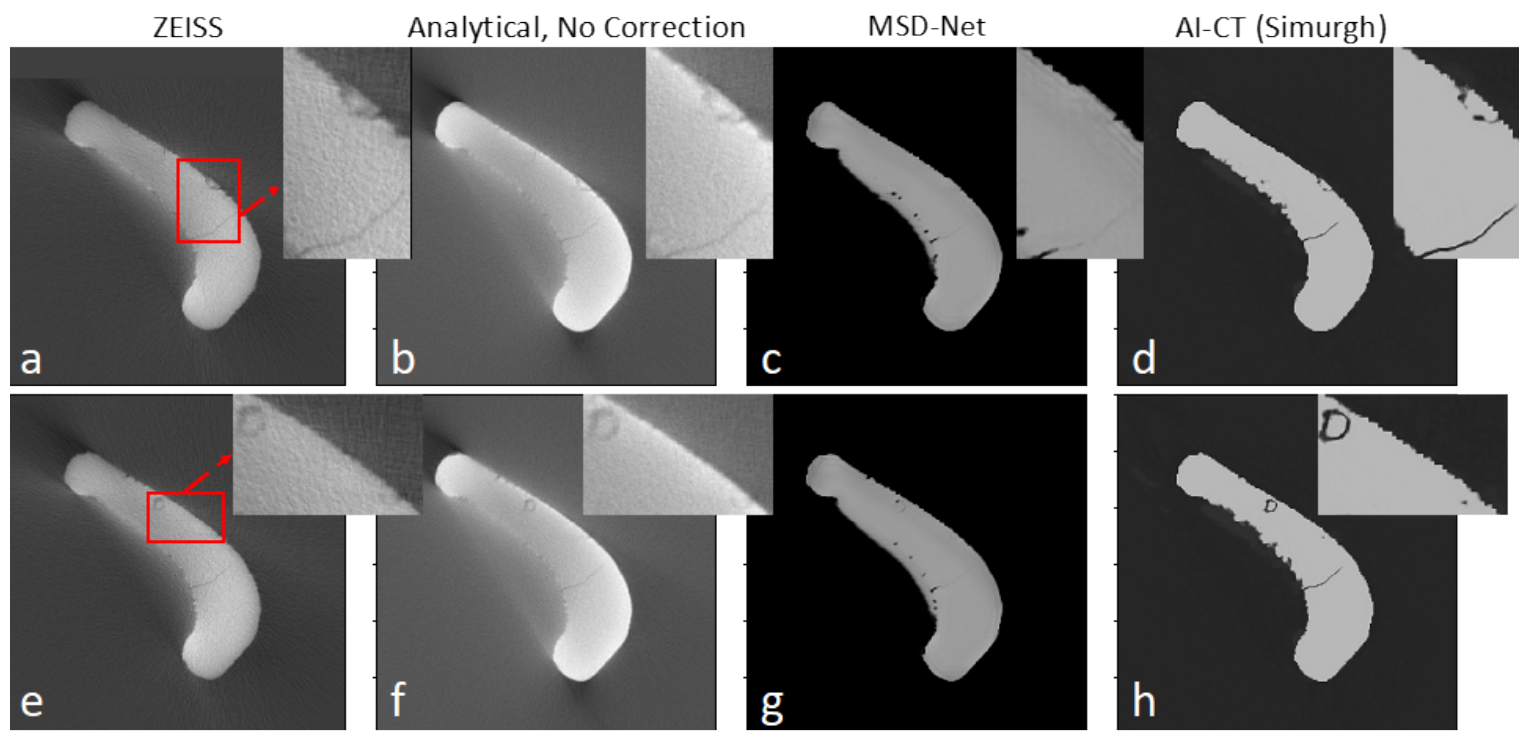

Figure A-14. Example test results on real data set. The proposed method is compared against the standard output from the ZEISS XCT system standard reconstruction, as well as the uncorrected standard analytical (FDK) approach and MSD-Net as a second neural network. Expanded views of areas of interest are highlighted in the inset images. 
Functionally, a convolutional neural network (denoted as $A I-C T$ in Figure A-12) is trained on the simulated (synthetic) data developed using CAD models and physics-based parameters. Then it is tested on synthetic and real data sets. Examples of tests on synthetic and real data sets are shown in Figure A-14. Two different neural networks - MSD-Net [3] and AI-CT [4], [5] — are tested to emphasize the modularity of this approach. The method is also compared against the standard output from the XCT system, as well as the uncorrected standard analytical (FDK) approach [6] and the state-of-the-art modelbased iterative reconstruction (MBIR) approach [7].

ORNL has published a peer-reviewed conference paper on this topic, and a provisional patent has been filed by ORNL. A Technology Innovation Program (TIP) proposal was submitted to the Technology Transfer Office, and since its acceptance, the full proposal is in development. The Simurgh method is supported by ZEISS Quality Solutions, and they are considering licensing the technology from ORNL. 


\section{A-9. REFERENCES}

[1] ASTM, "Standard Terminology for Additive Manufacturing." ISO / ASTM52900 - 15, 2015.

[2] L. Scime, D. Siddel, S. Baird, and V. Paquit, "Layer-Wise Anomaly Detection and Classification for Powder Bed Additive Manufacturing Processes: A Machine-Agnostic Algorithm for RealTime Pixel-Wise Semantic Segmentation,” Addit. Manuf., p. 101453, Jul. 2020.

[3] D. Pelt, K. Batenburg, and J. Sethian, "Improving Tomographic Reconstruction from Limited Data Using Mixed-Scale Dense Convolutional Neural Networks," J. Imaging, vol. 4, no. 11, p. 128, Oct. 2018.

[4] A. Ziabari, D. H. Ye, S. Srivastava, K. D. Sauer, J. B. Thibault, and C. A. Bouman, "2.5D Deep Learning for CT Image Reconstruction Using A Multi-GPU Implementation," Conf. Rec. Asilomar Conf. Signals, Syst. Comput., vol. 2018-Octob, pp. 2044-2049, 2019.

[5] A. Ziabari et al., "Beam Hardening Artifact Reduction in X-ray CT Reconstruction of 3D Printed Metal Parts Leveraging Deep Learning and CAD Models," in ASME IMECE 2020, 2020.

[6] L. A. Feldkamp, L. C. Davis, and J. W. Kress, "Practical Cone-Beam Algorithm," J. Opt. Soc. Am., pp. 612-619, 1984.

[7] P. Jin, C. A. Bouman, and K. D. Sauer, "A Model-Based Image Reconstruction Algorithm with Simultaneous Beam Hardening Correction for X-Ray CT," IEEE Trans. Comput. Imaging, vol. 1, no. 3, pp. 200-216, Sep. 2015. 